Aus der Klinik für Nephrologie und Rheumatologie

(Prof. Dr. med. G. A. Müller)

der Medizinischen Fakultät der Universität Göttingen

\title{
Point of care-Ultraschall versus Röntgen-Thorax zur Lagekontrolle zentralvenöser Katheter
}

\author{
INAUGURAL-DISSERTATION \\ zur Erlangung des Doktorgrades \\ der Medizinischen Fakultät der \\ Georg-August-Universität zu Göttingen
}

vorgelegt von

\section{Eirini Mavropoulou}

aus

Athen (Griechenland)

Göttingen 2017 
Referent :

Korreferent :
Prof. Dr. med. D. Patschan

PD. Dr. med. W. Staab 
Hiermit erkläre ich, die Dissertation mit dem Titel "Point of care-Ultraschall versus Röntgen-Thorax zur Lagekontrolle zentralvenöser Katheter " eigenständig angefertigt und keine anderen als die von mir angegebenen Quellen und Hilfsmittel verwendet zu haben.

Göttingen, den (Unterschrift) 


\section{Inhaltsverzeichnis}

Abbildungsverzeichnis ............................................................................ III

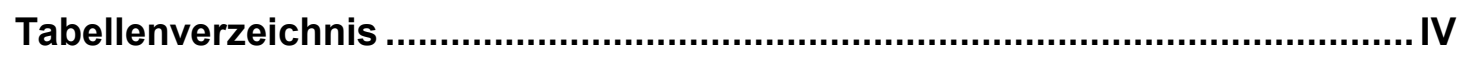

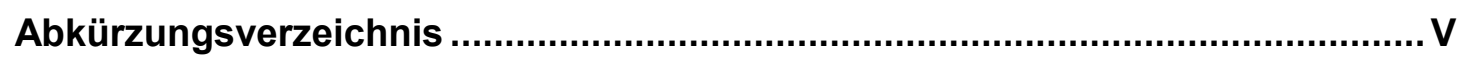

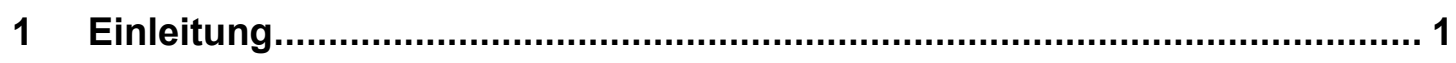

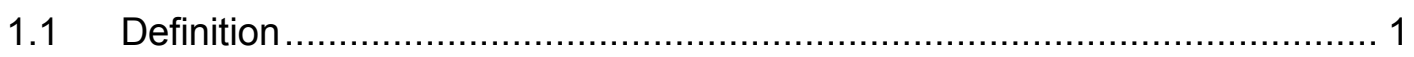

1.2 Historischer Rückblick .................................................................. 1

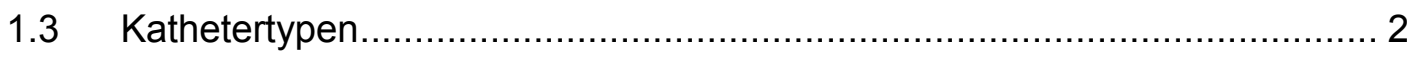

1.1.1 Nicht-getunnelte (temporäre) Katheter ......................................... 2

1.1.2 Getunnelte (langfristige) Katheter............................................. 2

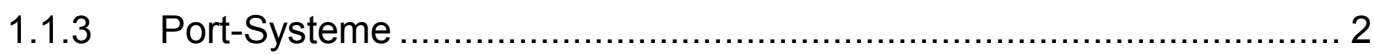

1.1.4 Peripherally inserted central venous catheters (PICC) ...................... 3

1.4 Zugangswege der zentralen Katheterisierung ...................................... 4

1.5 Indikationen und Kontraindikationen ................................................... 5

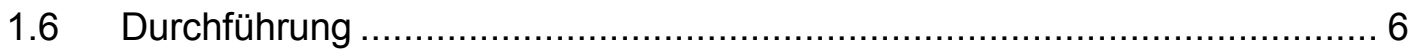

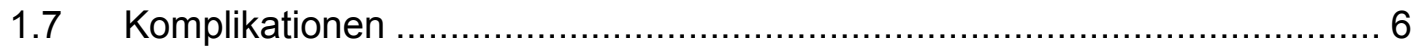

1.1.5 Periprozedurale Komplikationen................................................. 6

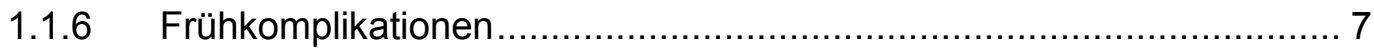

1.1.7 Spätkomplikationen................................................................ 7

1.8 Verwendete Techniken zur Lagekontrolle ............................................. 7

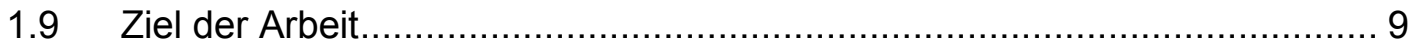

2 Patienten, Materialien und Methoden ........................................................ 11

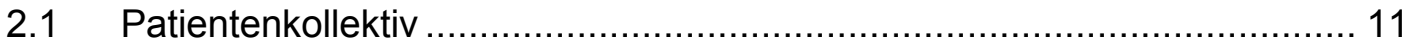

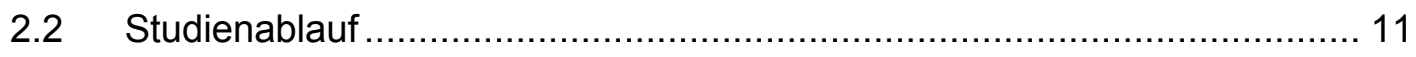

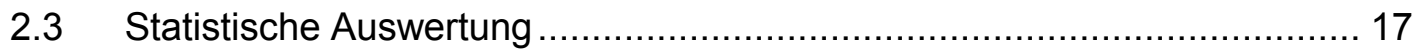

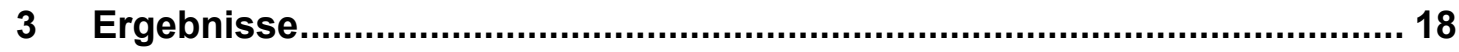

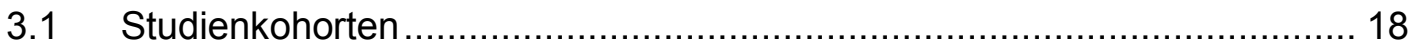

3.2 Analyse der postprozeduralen radiologischen Kontrolle ........................ 19

3.3 Das echokardiographische rapid atrial swirl sign - RASS ....................... 20

3.4 Testcharakteristika des rapid atrial swirl signs - RASS ........................... 21

3.5 Die interrater-Reliabilität des rapid atrial swirl signs - RASS ................... 21

3.6 Vergleich zwischen Echokardiographie und Röntgen ........................... 22

3.7 Der Ausbildungsgrad der Assistenzärzte........................................ 23 
3.8 Testcharakteristika des RASS in den verschiedenen Gruppen der

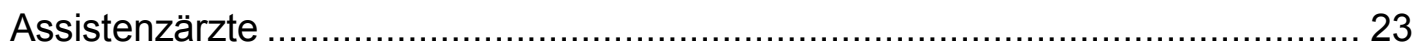

3.9 BMI und Dauer der Echokardiographie .............................................. 25

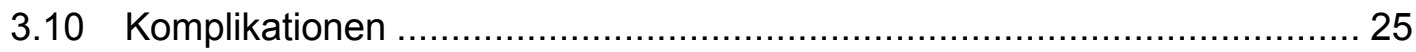

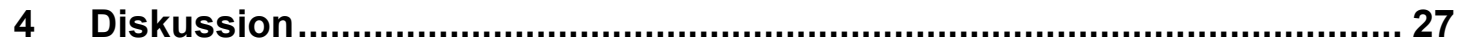

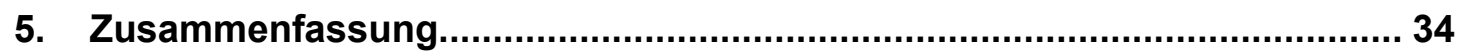

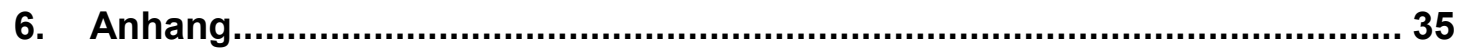

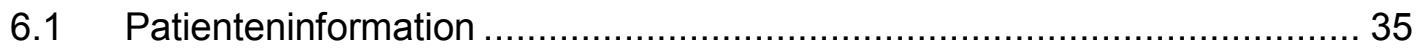

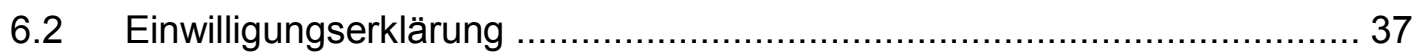

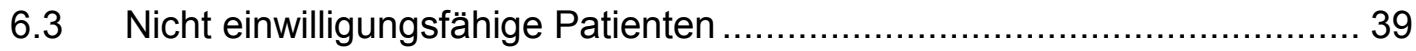

6.4 Wiedererlagung der Einwilligungsfähigkeit ............................................ 40

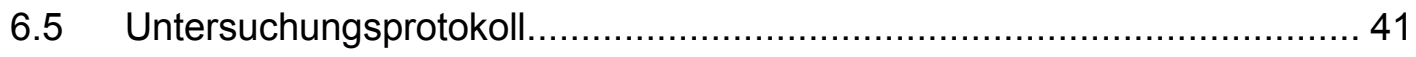

6.6 Einwilligungserklärung für Abbildungen............................................. 42

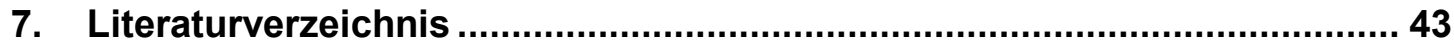




\section{Abbildungsverzeichnis}

Abb. 1: Applikation der Lokalanästhesie

Abb. 2: Punktion des Gefäßes unter Aspiration

Abb. 3: Einführung des Seldinger-Führungsdrahtes

Abb. 4: Dilatation des Stichkanals

Abb. 5: Entfernung des Führungsdrahtes und Einführung des Katheters.

Abb. 6: Durchführung der fokussierten Echokardiographie

Abb. 7: Demonstration des RASS in subkostalem Vierkammerblick

Abb. 8: Zeitlicher Ablauf der Studie

Abb. 9: Posterior - anterior - Röntgenaufnahme des Thorax nach ZVK-Anlage. 16

Abb. 10: Posterior - anterior - Röntgenaufnahme des Thorax nach ZVK-Anlage mit gezeichneter Linie durch die Carina

Abb. 11: Unterteilung der möglichen ZVK - Positionen in vier Zonen.

Abb. 12: Verteilung der Katheter-Lage in den vier radiologischen Zonen

Abb. 13: (A) Korrelation der Bewertung der Röntgenaufnahmen zwischen zwei unabhängigen Untersuchern mit linearer Regression. (B) Bland-Altman-Plot mit Darstellung der Differenz zum Mittelwert der Katheterposition in Relation zur Carina durch zwei Untersucher

Abb. 14: Die zeitliche Dauer für die Echokardiographie und das Röntgen in der Testund Validierungskohorte.

Abb. 15: Boxplot zur Darstellung des Einflusses des Weiterbildungsjahres auf die Dauer der Echokardiographie

Abb. 16: Streudiagramm zur Darstellung des Einflusses des BMI auf die Dauer der Echokardiographie

Abb. 17: Persistierende linke Vena cava superior im Röntgen-Thorax $(A)$ und im CT (B)

Abb. 18: Fehllage des zentralvenösen Katheters im rechten Ventrikel mit retrogradem flush. 


\section{Tabellenverzeichnis}

Tab. 1: Vergleichende Darstellung der gebräuchlichsten zentralvenösen Kathetersysteme

Tab. 2: Periprozedurale Komplikationen

Tab. 3: Frühkomplikationen

Tab. 4: Spätkomplikationen

Tab. 5: Charakteristika der Studienkohorten

Tab. 6: Ergebnisse der Röntgenaufnahmen und der Echokardiographie in beiden Kohorten

Tab. 7: Testcharakteristika des rapid atrial swirl signs in der Test- und Validierungskohorte

Tab. 8: Die interrater-Reliabilität bei der Bewertung des rapid atrial swirl signs mit drei Variablen

Tab. 9: Die interrater-Reliabilität bei der Bewertung des rapid atrial swirl signs mit zwei Variablen

Tab. 10: Zusammensetzung der Gruppen und mittlere Anzahl von Untersuchungen pro Assistenzarzt

Tab. 11: Testcharakteristika zwischen den verschiedenen Gruppen der Assistenzärzte

Tab. 12: Übersicht der publizierten Studien und ihrer Merkmale 29

Tab. 13: Ergebnisse der bereits publizierten Studien 


\section{Abkürzungsverzeichnis}

A. = Arteria

Abb. $=$ Abbildung

Abs. $=$ Absatz

a.p. = anterior-posterior

$\mathbf{B M I}=$ body mass index

B-Mode $=$ brightness modulation

$\mathbf{C T}=$ Computertomographie

CXR = chest $X$-ray, Röntgen-Thorax

EKG = Elektrokardiogramm

FFP = fresh frozen plasma, gefrorenes Frischplasma

ICU = Intensiv Care Unit, Intensivstation

IMC = Intermediate Care

IV = invasive ventilation, invasive Beatmung

$\mathbf{K I}=$ Konfidenzintervall

$\mathbf{L R}=$ Likelihood Ratio

$\max =$ maximum

MHz = Megahertz

Min. = Minute

$\min =\operatorname{minimum}$

MRT = Magnetresonanztomographie

ms $=$ Millisekunde

MTRA = medizinisch-technische/r Radiologieassistent/in

$\mathbf{N} / \mathbf{A}=$ not available

NIV = non-invasive ventilation, nicht invasive Beatmung 
NPV = Negative Predictive Value, negativer prädiktiver Wert

PACS = Picture Archiving and Communication System

PICC = Peripherally Inserted Central venous Catheters

PPV $=$ Positive Predictive Value, positiver prädiktiver Wert

RASS = Rapid Atrial Swirl Sign

SD = Standard Deviation, Standardabweichung

Sek. $=$ Sekunde

StrISchV = Strahlenschutzverordnung

TTE $=$ transthorakale Echokardiographie

TVT = tiefe Venenthrombose

US $=$ Ultraschall

V. = Vena

Vv. = Venae

ZVD = zentralvenöser Druck

ZVK = zentraler Venenkatheter 


\section{$1 \quad$ Einleitung}

\subsection{Definition}

Ein zentraler Venenkatheter (ZVK) ist ein Katheter mit Spitze, welcher im proximalen Drittel der Vena cava superior, im rechten Vorhof oder in der Vena cava inferior platziert wird. Der entsprechende Katheter kann entweder durch eine periphere Vene oder eine proximal gelegene zentrale Vene, meistens die jugularis interna, subclavia oder femoralis eingelegt werden (Smith und Nolan 2013). Der ZVK ermöglicht einen zuverlässigen intravenösen Zugang für Blutentnahmen, die Gabe von Medikamenten sowie ggf. eine parenterale Ernährung (Baskin et al. 2009). In den USA werden mehr als 5 Millionen zentralvenöse Katheter jährlich angelegt (Raad 1998), in Deutschland ca. 1,9 Millionen (Schummer et al. 2005).

\subsection{Historischer Rückblick}

Die erste Veröffentlichung einer Katheterisierung von zentralen Gefäßen datiert auf das Jahr 1733 vom englischen Geistlichen Stephen Hales, der den arteriellen und venösen Blutdruck eines Pferdes bestimmen wollte (Hales 1733). Claude Bernard führte 1844 in Frankreich die erste Herzkatheterisierung über die Arteria carotis und die Vena jugularis interna eines Pferdes durch. Das Ziel bestand hierbei in der Temperaturmessung im rechten und linken Ventrikel. Im Jahr 1876 wurde im Rahmen einer Autopsie eines Hundes als erste Komplikation der zentralvenösen Katheterisierung die Perforation des linken Ventrikels mit begleitender perikardialer Hämorrhagie (Bernard 1876) berichtet. Bleichröder unternahm im Jahr 1905 die ersten Experimente zur Katheteranlage in Arterien und Venen am Menschen, die Untersuchungen wurden jedoch erst 1912 publiziert (Bleichroeder et al. 1912).

Ein erster Pionier in der zentralvenösen sowie kardialen Katheterisierung war Werner Forssmann, ein 25-jähriger Assistenzarzt aus Eberswalde. Nach der Ablehnung der Durchführung von Experimenten an Patienten durch seinen Chefarzt unternahm er im Sommer 1929 einen Selbstversuch. Dabei betäubte er seine linke Ellenbeuge und schob anschließend einen $65 \mathrm{~cm}$ langen Urinkatheter bis zum rechten Herzen vor. Anschließend stieg er die Treppe mit liegendem Katheter bis zum Röntgen-Raum hinauf, wo er die Position des Katheters radiologisch darstellte (Forssmann 1929). In den folgenden zwei Jahren experimentierte er weiter und zeigte, dass diese Katheter die Applikation von verschiedenen Medikamenten und Kontrastmitteln ermöglichen (Forssmann 1931). Er erhielt für seine Arbeiten im Jahr 1956 den Nobelpreis für Medizin.

Meyers und Zimmermann verwendeten 1945 flexible Katheter aus Polyurethan, um Kindern Flüssigkeit und parenterale Ernährung zu verabreichen (Meyers 1945; Zimmermann 1945). 1952 beschrieb der französische Militärarzt Aubaniac als erster die Punktionstechnik der Vena subclavia, um eine forcierte Flüssigkeitssubstitution bei Kriegsverletzten in Vietnam zu ermöglichen (Aubaniac 1952). Eine ausschlaggebende technische Weiterentwicklung erfolgte 1953 durch den schwedischen Radiologen Sven-Ivar Seldinger, der eine neue Methode zur Katheterisierung mittels Führungsdraht veröffentlichte (Seldinger 1953). Die Seldinger-Technik gilt heutezutage als Standardtechnik. 
In den folgenden Jahren kam es zu einer Verbreitung der zentralvenösen Katheterisierung mit gleichzeitiger Zunahme von Komplikationen, vor allem aufgrund der anatomisch orientierten Punktionstechniken (engl. landmark method). Daraufhin wurden im Jahr 1982 und 1984 die ersten Studien zur ultraschall-gesteuerten Punktion publiziert, welche eine signifikante Risikoreduktion belegen konnten (Peters et al. 1982; Legler und Nugent 1984).

Die optimale Methode zur Lagekontrolle des zentralvenösen Katheters ist bis heute ein wichtiges und kontroverses Thema. Die aktuelle Standardmethode ist die konventionelle Röntgenaufnahme des Thorax, wobei die korrekte Lage als Position der Katheterspitze in der Vena cava superior, d. h. auf Höhe der Carina definiert wird (Schuster et al. 2000). Es wurden mehreren Alternativen gesucht, wie zum Beispiel die Verwendung von Ultraschall bei Neugeborenen (Ohki et al. 2000), die transösophageale Echokardiographie (Reynolds et al. 2001) oder die Positionierung mit Hilfe der intrakardialen EKG-Ableitung (Hellerstein et al. 1949). Keines der benannten Alternativverfahren ist bislang als gleichwertig etabliert.

\subsection{Kathetertypen}

Eine große Vielzahl von Kathetersystemen bzw. -typen deckt die verschiedenen Indikationen zur zentralvenösen Katheterisierung ab. Die vier Hauptkategorien zentralvenöser Katheter sind: nicht-getunnelte (temporäre) und getunnelte (langfristige) Katheter, Port-Systeme und so genannte peripherally inserted central venous catheters (PICC).

\subsubsection{Nicht-getunnelte (temporäre) Katheter}

Bei den nicht-getunnelten Kathetern befindet sich die Einstichstelle in unmittelbarer Nähe zum Gefäß. In diese Gruppe gehören der ein- bis fünflumige zentralvenöse Katheter (ZVK) und der hauptsächlich zur Hämodialyse verwendete großlumige Shaldon-Katheter mit zwei oder drei Lumen. Die verwendeten Materialien sind überwiegend aus Silikon (weich) und Polyurethan (steif). Die häufigste Komplikation ist eine Infektion durch Keimbesiedlung, so dass bei einer Anlagedauer von mehr als 14 Tagen ein getunnelter Dialysekatheter zu bevorzugen ist (Weijmer et al. 2004). Bei ZVKs erfolgt bei einer vermuteten Katheterinfektion der Wechsel des Katheters.

\subsubsection{Getunnelte (langfristige) Katheter}

Das Charakteristikum getunnelter Katheter ist ein subkutaner Verlauf in der Thoraxwand für einige cm (ca. 8-15 cm), bevor dieser ins Gefäß eintritt. Eine im subkutanen Verlauf befindliche Dacron-Muffe dient als zusätzliche Barriere gegenüber Infektionen. In einer Metaanalyse zeigten die getunnelten Katheter mit Muffe deutlich geringere Infektionsraten als ohne eine solche (Maki et al. 2006). Als Materialien werden dünnes Polyurethan oder Silikon bevorzugt, welche lokal weniger traumatisierend wirken. Die bekanntesten Vertreter dieser Gruppe sind der Hickman-, Broviac-, Demers-, Groshong- und Leonard-Katheter.

\subsubsection{Port-Systeme}

Das implantierte Portsystem besteht aus einer Kammer, die mit einem Katheter verbunden ist. Die Kammer wird operativ direkt in die Subcutis eingesetzt und hat eine 
kräftige Silikonmembran, welche mit Titan oder Plastik befestigt ist. Sie kann ca. zweitausendmal mit einer speziellen Portkanüle durchstochen werden. Der intravenöse Katheter ist ähnlich beschaffen wie ein ZVK und wird in das zentralvenöse Gefäß eingebracht. Portsysteme minimieren das Risiko einer systemischen Infektion und ermöglichen eine aktive Teilnahme an Sport und Freizeit. Der große Nachteil besteht in Katheterokklusionen, meistens durch Thrombosierungen (Walser 2012). In diesen Fällen ist oftmals ein operativer Austausch unumgänglich.

\subsubsection{Peripherally inserted central venous catheters (PICC)}

Der PICC besteht ebenfalls aus Silikon oder Polyurethan, ist 3 - 4 French groß und verfügt über ein, zwei oder drei Lumen. Der Katheter wird über eine periphere Vene des Armes (V. cephalica, V. basilica, V. brachialis) eingeführt, und seine Spitze wird unter Röntgendurchleuchtung in der $V$. cava superior positioniert. Die einfache und komplikationsarme Anlage ist vorteilhaft, es kommt aufgrund des vergleichsweise kleinen Durchmessers jedoch häufig zu Okklusionen durch Thrombosierung. Das Risiko einer systemischen Infektion ist bei PICCs und bei den nicht-getunnelten zentralvenösen Kathetern bei hospitalisierten Patienten vergleichbar (Maki et al. 2006; Chopra et al. 2013b). In einer Metaanalyse der Ergebnisse aus 64 Studien mit 29.503 Patienten waren die PICCs im Vergleich zu anderen ZVKs mit einem erhöhten Risiko für tiefe Venenthrombosen, nicht jedoch für Lungenarterienembolien assoziiert. Patienten mit schweren Erkrankungen oder malignen Tumoren waren besonders gefährdet (Chopra et al. 2013a).

Tabelle 1 fasst die benannte Kathetertypen sowie die mit deren Einsatz verbundenen Vor- und Nachteile zusammen.

Tab. 1: Vergleichende Darstellung der gebräuchlichsten zentralvenösen Kathetersysteme (CLABSI Toolkit - Chapter 1 | Joint Commission).

Verwendete Symbole und Abkürzungen: kurz*: weniger als drei Wochen, lang†: Wochen bis Monate, PICC: Peripherally Inserted Central venous Catheters, TVT: tiefe Venenthrombose.

\begin{tabular}{|c|c|c|c|c|}
\hline Katheter-Typ & Zugangsweg & $\begin{array}{l}\text { Liege- } \\
\text { dauer }\end{array}$ & Vorteile & Nachteile \\
\hline nicht getunnelt & $\begin{array}{l}\text { - } \text { V. jugularis interna } \\
\text { - } \quad \text { V.subclavia } \\
\text { - } \quad \text { V. femoralis }\end{array}$ & kurz ${ }^{*}$ & $\begin{array}{ll}\text { - } & \text { einfache Insertion } \\
\text { - } & \text { günstig } \\
\text { - } & \text { wenige Komplikationen }\end{array}$ & $\begin{array}{ll}\text { - } & \text { häufig Okklusion } \\
\text { - } & \text { Verletzung der beiliegen- } \\
& \text { den Gewebe } \\
\text { - } & \text { Infektionsrisiko }\end{array}$ \\
\hline getunnelt & $\begin{array}{ll}\text { - } & \text { V. jugularis interna } \\
\text { - } & \text { V. subclavia } \\
\text { - } & \text { V. femoralis }\end{array}$ & lang† & $\begin{array}{l}\text { - } \quad \text { weniger Okklusion } \\
\text { - } \quad \text { weniger Infektionen }\end{array}$ & $\begin{array}{l}\text { - } \text { erhöhte Kosten } \\
\text { - } \text { benötigt operativen Be- } \\
\text { dingungen }\end{array}$ \\
\hline Ports & $\begin{array}{l}\text { - } \text { V. jugularis interna } \\
\text { - } \quad \text { V. subclavia }\end{array}$ & lang† & $\begin{array}{ll} & \text { wenig sichtbar } \\
\text { - } & \text { weniger Infektionen } \\
\text { - } & \text { bessere Lebensqualität }\end{array}$ & $\begin{array}{ll}\text { - } & \text { erhöhte Kosten } \\
\text { - } & \text { benötigt operativen Be- } \\
\text { dingungen } \\
\text { - Okklusion } \\
\text { - } \text { Paravasation }\end{array}$ \\
\hline PICC & $\begin{array}{ll}\text { - } & \text { V. cephalica } \\
\text { - } & \text { V. basilica } \\
\text { - } & \text { V. brachialis }\end{array}$ & kurz* & $\begin{array}{l}\text { - } \text { einfache Anlage (bedside) } \\
\text { - günstig und sicher }\end{array}$ & $\begin{array}{ll}\text { - } & \text { häufig Okklusion } \\
\text { - } & \text { evtl. erschwerte Insertion } \\
\text { in die Vena cava superior } \\
\text { - } \quad \text { TVT }\end{array}$ \\
\hline
\end{tabular}




\subsection{Zugangswege der zentralen Katheterisierung}

Die Auswahl des Zugangsweges basiert auf verschiedenen Faktoren:

- Indikation und voraussichtliche Liegedauer des zentralvenösen Katheters

- Anatomie und klinischer Zustand des Patienten

- Erfahrung/Präferenz des Punkteurs

- mögliche Komplikationen oder Risiken

Der bevorzugte Zugangsweg ist die $\mathrm{V}$. jugularis interna, die lateral von der Trachea in der Vagina carotica zusammen mit der A. carotis communis und dem Nervus vagus verläuft (Waldeyer 2003). Sie kann mit Hilfe von anatomischen Landmarken, des Pulses der A. carotis oder sonographisch detektiert werden. Der gerade Verlauf zur V. cava superior erleichtert das Vorschieben des Führungsdrahtes und des Katheters. Entscheidender Nachteil ist die Gefahr einer akzidentellen Punktion der A. carotis mit anschließender Blutung, Dissektion oder Apoplex durch die Verschleppung von Thromben in die zerebralen Gefäße (Guilbert et al. 2008). Zusätzlich besteht ein höheres Risiko von katheterassoziierten Infektionen im Vergleich zur V. subclavia, wahrscheinlich aufgrund der unmittelbaren Nähe zu oropharyngealen Sekreten, der lokal höheren Temperatur und der erschwerten Fixierung des Verbandmaterials um die Einstichsstelle (Moro et al. 1994; Parienti et al. 2012).

Ein ebenfalls verbreiteter Zugangsweg ist die V. subclavia, welche in Höhe der 1. Rippe aus der $V$. axillaris hervorgeht und sich hinter dem Sternoklavikulargelenk mit der V. jugularis interna zur V. brachiocephalica vereinigt (Frick et al. 1992). Ein Vorteil des Subclaviazuganges ist die einfache Punktion angesichts klarer anatomischer Orientierungsstrukturen und des guten Füllungszustandes des Gefäßes, auch in Schockzuständen. Die Katheterisierung der V. subclavia zeigt ein signifikant geringeres Risiko für katheterassoziierte Infektionen dank niedrigerer lokaler Keimzahl und größerer Distanz zwischen der Einstichsstelle und der Eintrittsstelle ins Gefäß (Parienti et al. 2015). Ein erheblicher Nachteil ist eine potentiell unkontrollierbare Blutung mit möglichem Hämatothorax im Falle einer arteriellen Fehlpunktion. Hier bestehen lokal schlechte Kompressionsmöglichkeiten mit einer Blutungsinzidenz von 0,1\% bis $1 \%$ (Kusminsky 2007). Die Verwendung langfristiger, getunnelter Katheter über die V. subclavia kann zudem eine Stenose des Gefäßes hervorrufen, wahrscheinlich bedingt durch die konstante Verletzung des Endothels, diese wurde in $20 \%$ bis $50 \%$ bei dialysepflichtigen Patienten beschrieben (Vats 2012). Das Risiko eines Pneumothorax ist bei der Katheterisierung der V. subclavia höher (McGee und Gould 2003). In einer systematischen Übersicht von zehn Studien mit insgesamt 3.420 Kathetern schien es keinen Unterschied für die Entstehung eines Pneumothorax zwischen V. jugularis und V. subclavia zu geben, in den ausgewählten Studien erfolgte jedoch keine Randomisierung, was potentiell zu einem Selektionbias geführt hat (Ruesch et al. 2002).

Die V. femoralis zieht medial von der A. femoralis durch die Lacuna vasorum und geht proximal vom Leistenband in die V. iliaca externa über (Waldeyer 2003). Die Katheterisierung der $\mathrm{V}$. femoralis wird meistens in Nofallsituationen benutzt. Die einfachen anatomischen Landmarken, die tastbare Pulsation der A. femoralis und die 
sofortige Verwendbarkeit des Katheters auch ohne radiologische Lagekontrolle ermöglichen eine schnelle Anlage mit geringem Blutungsrisiko. Es gibt Kontroversen bzgl. der Inzidenz von katheterassoziierten Infektionen zwischen den drei Zugangswegen. In einer Metaanalyse verschiedener randomisierter, kontrollierter und Kohortenstudien wurde kein signifikanter Unterschied hinsichtlich des Infektionsrisikos beschrieben (Marik et al. 2012). In einer großen multizentrischen Studie an 3.027 Patienten war das Risiko einer katheterassoziierten Sepsis in der Femoralis-Gruppe signifikant höher als in der Subclavia-Gruppe, wobei zwischen Femoralis- und Jugularis-Gruppe kein Unterschied bestand. Zusätzlich wurden statistisch signifikant häufigere Thrombosen im Vergleich zu den Vv. jugularis und subclavia beschrieben (Parienti et al. 2015).

\subsection{Indikationen und Kontraindikationen}

Die Indikationen für zentralvenöse Katheterisierung sind (Lang 2012; Ingrande und Lemmens 2014):

- Infusion von Lösungen, die stark venenreizend wirken, wie:

- Zytostatika

- $\quad$ einige Antibiotika ( $\mathrm{pH}-$ Wert >9)

- höher dosiertes Natriumbikarbonat

- Kaliumchlorid

- hochkalorische Glucose- oder Aminosäureninfusionen, vor allem bei parenteraler Ernährung

- Infusion von kreislauf- und herzwirksamen Medikamenten mit kurzer Halbwertszeit, zum Beispiel Katecholamine

- Langzeit-Infusionstherapie (> 10 Tage)

- Infusion durch großlumige Katheter, sofern kein peripherer Zugang möglich ist, zum Beispiel bei

- sämtlichen Schockformen (vor allem Volumenmangelschock, septischer Schock)

- Hypothermie

- ausgedehnten Verbrennungen

- hämodynamische Überwachung

- Messung des zentralen Venendrucks

- zentralvenöse Sättigung

- Beurteilung des intravasalen Volumenstatus und der rechtsventrikulären Funktion

- venöse Hämofiltration und -dialyse (zum Beispiel mehrlumige Shaldon-Katheter)

- Interventionen

- temporärer Schrittmacher

- intravaskuläres Temperaturmanagement

- wiederholte Blutentnahmen

- Therapie einer venösen Luftembolie zum Beispiel während neurochirurgischer Eingriffe

Es gibt relative Kontraindikationen, die jedoch abhängig sind von der Dringlichkeit und den Alternativen für einen venösen Zugang (McGee und Gould 2003): 
- anatomische Fehlbildung, beispielsweise lokale Infektionen der Haut oder Narbengewebe

- Koagulopathien und Thrombozytopenie

- Studien zeigten, dass schwere Blutungen nach ZVK-Anlage selten sind (Kander et al. 2013) und dass die prophylaktische Gabe von FFPs keinen signifikanten Unterschied hinsichtlich des Risikos einer Blutungskomplikation bewirkt (Müller et al. 2015)

- ZVK-Anlage kann ab einer Thrombozytenzahl von > $20 \times 10^{9} / \mathrm{l}$ gefahrlos durchgeführt werden (Zeidler et al. 2011)

- die Thrombozytopenie ist ein größerer Risikofaktor für Blutungen als eine verlängerte Koagulationszeit (Polderman und Girbes 2002)

- es ist allgemein empfohlen, dass eine Katheterisierung der Vena subclavia aufgrund fehlender Komprimierbarkeit des Gefässes bei Gerinnungsstörungen vermieden werden sollte

- Allergie des Patienten auf die Materialien des ZVK

- ipsilateraler Pneumothorax oder Hämatothorax

- Thrombose, Stenose oder andere anatomische Veränderung des Gefäßes

- ipsilateral liegende intravaskuläre Aggregate, zum Beispiel SchrittmacherAggregate

\subsection{Durchführung}

Die detailierte Beschreibung der Durchführung einer ZVK-Anlage erfolgt im Methodikteil.

\subsection{Komplikationen}

Die Komplikationen der ZVK-Anlage sind vielfältig und hängen wesentlich von den anatomische Gegebenheiten und der Erfahrung des ausführenden Arztes ab. In einer Übersichtsarbeit wurde eine gesamte Komplikationsrate von $15 \%$ beschrieben (McGee und Gould 2003).

Die Komplikationen werden in drei Kategorien unterteilt (Teichgräber et al. 2004).

\subsubsection{Periprozedurale Komplikationen}

Die periprozedurale Komplikationen finden bei der Implantation statt.

Tab. 2: Periprozedurale Komplikationen.

Periprozedurale Komplikationen

\begin{tabular}{|l|}
\hline Arterielle Fehlpunktion \\
\hline Hämatom \\
\hline Luftembolie \\
\hline Pneumothorax \\
\hline Katheterfehllage \\
\hline Plexusirritation \\
\hline Perforation \\
\hline - Vena cava superior \\
\hline$-\quad$ Herzwand \\
\hline$-\quad$ Pulmonalarterie \\
\hline
\end{tabular}




\subsubsection{Frühkomplikationen}

Die Frühkomplikationen treten direkt im Anschluss an und bis zu eine Woche nach Katheterinsertion auf (Morano et al. 2014).

Tab. 3: Frühkomplikationen.

Frühkomplikationen

\begin{tabular}{|l|}
\hline Hämatom \\
\hline Luftembolie \\
\hline Pneumothorax \\
\hline Infektion \\
\hline Venenthrombose \\
\hline Katheterfehllage \\
\hline Katheterdysfunktion, Okklusion \\
\hline Arteriovenöse Fistel \\
\hline Perforation \\
\hline - Vena cava superior \\
\hline - Herzwand \\
\hline$-\quad$ Pulmonalarterie \\
\hline
\end{tabular}

\subsubsection{Spätkomplikationen}

Die Spätkomplikationen treten häufiger bei im Verlauf nicht fachgerechter Versorgung des ZVK auf.

Tab. 4: Spätkomplikationen.

Spätkomplikationen

\begin{tabular}{|l|}
\hline Luftembolie \\
\hline Infektion \\
\hline Venenthrombose \\
\hline Katheterfehllage \\
\hline Katheterdysfunktion, Okklusion \\
\hline Arteriovenöse Fistel \\
\hline Perforation \\
\hline$-\quad$ Vena cava superior \\
\hline$-\quad$ Herzwand \\
\hline$-\quad$ Pulmonalarterie \\
\hline pinch-off-Katheterbruch \\
\hline Kathetermigration \\
\hline
\end{tabular}

\subsection{Verwendete Techniken zur Lagekontrolle}

Zur Erkennung und Prävention von Komplikationen der zentralvenösen Katheteranlage ist eine Lagekontrolle unentbehrlich. Die Spitze des Katheters sollte oberhalb der Mündung der Vena cava superior mit dem rechten Vorhof und außerhalb des Perikardiums liegen (Hsu et al. 2006; Smith und Nolan 2013). Zusätzlich soll sie parallel mit dem Gefäß verlaufen, sodass kein Kontakt mit dem Endothel oder dem Endokard besteht (Fletcher und Bodenham 2000). Das Perforationsrisiko steigt an, 
wenn der Winkel zwischen Katheter und Gefäßwand größer als $40^{\circ}$ ist (Gravenstein und Blackshear 1991; Walshe et al. 2007).

Bisher existiert jedoch kein etabliertes Verfahren, das alle Charakteristika erfüllt. Eine Möglichkeit ist die Überprüfung der freien Aspirierbarkeit von Blut über alle Schenkel des Katheters oder der Ausschluss einer intraarteriellen Lage mittels Blutgasanalyse während der Anlage. Eine dritte Möglichkeit ist es, mit Hilfe einer invasiven Blutdruckmessung über einen Schenkel des ZVK durch respiratorische Fluktuationen der Druckkurve die intravenöse Lage zu bestätigen (Losert et al. 2000; Schummer et al. 2001).

Eine weitere, häufig verwendete Methode ist die elektrokardiographisch-gestützte Positionsbestimmung, die 1949 von Hellerstein erstbeschrieben wurde (Hellerstein et al. 1949). Hierbei wird eine EKG-Ableitung über die Katheterspitze durchgeführt. Die Amplitude der p-Welle, die der elektrischen Erregung des rechten Vorhofs entspricht, verändert sich mit Vorschub in den rechten Vorhof und zeigt eine maximale Erhöhung auf Höhe der Crista terminalis (Schummer et al. 2005). Anschließend wird der Katheter zurückgezogen, bis die p-Welle sich wieder normalisiert. Mit dem Verfahren konnte eine Verbesserung in der Positionierung der zentralvenösen Katheter mit weniger Zeitverzögerung, Senkung der Kosten und fehlender Strahlenbelastung erreicht werden (David et al. 2005; Gebhard et al. 2007; Pittiruti et al. 2012). Eine wichtige Einschränkung sind allerdings mögliche Herzrhythmusstörungen und Bewegungsartefakte, die die Beurteilung des EKGs erschweren sowie die Gefahr, dass der von links eingeführte Katheter in einem ungünstigen Winkel zur Venenwand liegt (Schummer et al. 2005). Ein weiterer Nachteil dieser Methode ist die fehlende Unterscheidbarkeit zwischen einem intravenösen und intraarteriellen EKG, was die Detektion einer arteriellen Fehllage unmöglich macht (Schummer et al. 2004).

Zwei weitere Methoden sind die Fluoroskopie und die transösophageale Echokardiographie, die eine sofortige Erkennung der korrekten Position ermöglichen. Beide Untersuchungen sind jedoch technisch aufwendiger und verhältnismäßig teuer, so dass sie vorwiegend für die Anlage langfristiger Katheter verwendet werden (Reynolds et al. 2001; Chaney et al. 2007; Ahn et al. 2012).

Das am häufigsten verwendete Verfahren ist die postprozedurale radiologische Kontrolle des Katheters mittels konventioneller Röntgenaufnahme des Thorax. In vielen Kliniken ist die Beurteilung der Röntgenaufnahme vor Verwendung des ZVK obligat, was jedoch unter Umständen eine große zeitliche Verzögerung der Therapie des Patienten nach sich zieht. Die Mündung der V. cava superior in das rechte Atrium ist radiologisch nicht genau erkennbar. In Untersuchungen an 34 Leichen zeigten Schuster et al., dass die Carina tracheae die beste radiologische Landmarke ist und das Perikardium immer unterhalb der Carina beginnt (Schuster et al. 2000). Dieser Befund wurde von Albrecht et al., die 39 Leichen untersuchten, bestätigt (Albrecht et al. 2004). In einer Publikation von Aslamy et al. wurde mittels MRT des Thorax von 42 Patienten gezeigt, dass die Länge der V. cava superior stark variiert von 4,4 bis $10 \mathrm{~cm}$ und dass der rechte tracheobronchiale Winkel immer mindestens $2,9 \mathrm{~cm}$ oberhalb des Übergangs zum rechten Vorhof liegt, deshalb empfiehlt er dies als die verlässlichste radiologische Landmarke (Aslamy et al. 1998). Ein großer Nachteil ist das schwierige Auffinden des Winkels, der nur in $32 \%$ aller Röntgenaufnahmen 
überhaupt detektiert werden kann. Die Carina kann wiederum in $96 \%$ identifiziert werden (Wirsing et al. 2008).

Der Frage nach der Notwendigkeit der Röntgenaufnahmen wurde in mehreren Studien nachgegangen. Mit Hilfe der transösophagealen Echokardiographie wurde zum Beispiel gezeigt, dass das Röntgen-Thorax eine intraatriale Position der Spitze nicht zuverlässig detektieren kann (Chu et al. 2004; Hsu et al. 2006; Wirsing et al. 2008). Bailey et al. berichteten, dass für Patienten unter EKG-Monitoring in einer IMC- oder ICU-Station das postprozedurale Röntgen unnötig ist, wenn der ZVK ohne Schwierigkeiten platziert wurde und weniger als drei Punktionsversuche erfolgten (Bailey et al. 2000). Andere Autoren empfehlen daher die Durchführungen des Röntgens nur, wenn klare Komplikationen vermutet werden (Gladwin et al. 1999; Molgaard et al. 2004).

In den letzten Jahren wurden wenige Studien publiziert, die sich mit der Frage beschäftigten, ob die Ultraschalluntersuchung am Patientenbett geeignet ist für die Lagekontrolle zentralvenöser Katheter und möglicherweise die Röntgenkontrolle gänzlich ersetzen kann. Initial wurde die sonographische Kontrolle der V. jugularis, V. subclavia und des Herzens zur Detektion der ZVK-Spitze beschrieben (Maury et al. 2001; Matsushima und Frankel 2010; Zanobetti et al. 2013). Der transthorakale Ultraschall der $V$. cava superior und des rechten Atriums hat jedoch eine eingeschränkte Qualität. Daraufhin entwickelten Vezzani et al. ein neues Verfahren, nämlich die Durchführung einer Echokardiographie mit gleichzeitiger Verabreichung von $9 \mathrm{ml}$ Kochsalzlösung zuzüglich $1 \mathrm{ml}$ Luft über den Katheter. Bei korrekter Lage kam es innerhalb von zwei Sekunden nach Injektion zu einer Verwirbelung (sog. flush oder rapid atrial swirl sign [RASS]) im rechten Vorhof und Ventrikel. Für diese Methode wurden eine Sensitivität von $96 \%$ und eine Spezifität von $93 \%$ beschrieben. Bezüglich des Zeitfaktors gab es eine signifikante Differenz von 73 Minuten zu Gunsten der Ultraschallmethode, was bei Intensivpatienten eine schnellere Nutzung zentralvenös zu applizierender Pharmaka (vor allem Katecholamine, größere Mengen Flüssigkeit) bedeuten kann (Vezzani et al. 2010). In den nächsten Jahren folgten weitere Studien (Campo Dell Orto et al. 2013; Wen et al. 2014; Meggiolaro et al. 2015) auch mit Applikation von reiner Kochsalzlösung (Liu und Bahl 2011; Weekes et al. 2014; DuranGehring et al. 2015; Gekle et al. 2015; Weekes et al. 2015), die die Effizienz der Methode verstärkten. Die fehlende Strahlenbelastung und die geringere Kosten sind zusätzliche Vorteile des Ultraschalls im Vergleich zum Röntgen.

\subsection{Ziel der Arbeit}

Zur Lagekontrolle von ZVK sind Röntgenaufnahmen des Thorax aktuell das Standardverfahren, weisen jedoch oben genannte Nachteile auf. Die transthorakale Echokardiographie mit Kochsalz-Injektion über den ZVK stellt eine neuartige Methode dar, welche eine korrekte Position oder eine Fehllage der Katheter-Spitze nachweisen kann. Um beide Verfahren zu vergleichen, führten wir eine prospektive, monozentrische klinische Studie in der Universitätsmedizin Göttingen durch. Der primäre Endpunkt war die Überprüfung der Sensitivität und Spezifität der fokussierten Echokardiographie im Vergleich zum konventionellen Röntgen-Thorax als Referenzmethode. Als sekundäre Endpunkte wurden die Dauer der jeweiligen Untersuchung, die Leistungsniveau der diensthabenden Ärzte für die Durchführung der Ultraschall- 
untersuchung geprüft und der Einfluss des Body-Mass-Indexes des Patienten auf die Dauer der Echokardiographie untersucht.

Die Ziele dieser Arbeit sind:

- die Effizienz und Sicherheit dieser Methode zu evaluieren

- den zeitlichen Unterschied zwischen Ultraschall und Röntgen zu vergleichen

- zu analysieren, ob der Ausbildungsstand des Untersuchers eine Rolle in der Bewertung der Ergebnisse spielt.

- zu analysieren, ob der Zeitbedarf für die Untersuchung durch den Ausbildungsstand des Untersuchers beeinflusst wird.

- zu analysieren, ob die Dauer der Echokardiographie mit dem BMI des Patienten korreliert. 


\section{Patienten, Materialien und Methoden}

\subsection{Patientenkollektiv}

Seitens der Ethikkommission der Universitätsmedizin Göttingen bestanden keine ethischen Bedenken gegen die Durchführung unseres Forschungsvorhabens (Antragsnummer 11/3/14). Die Studie wurde zudem unter www.clinicaltrials.gov (Nummer NCT02661607) registriert.

Im Rahmen unserer Studie wurden insgesamt 100 Patienten auf der internistischen Intermediate Care Station (IMC-Station 1021) sowie der nephrologischen Intensivstation (ICU-Station 1022) der Klinik für Nephrologie und Rheumatologie vom November 2014 bis April 2016 prospektiv eingeschlossen. Ein screening bzw. Einschluss erfolgte, wenn aus medizinischer Sicht die Indikation zur Versorgung mit einem zentralvenösen Katheter bestand.

Die Einschlusskriterien waren:

1. Volljährigkeit

2. gute Kenntnisse der deutschen Sprache

3. medizinische Notwendigkeit für die Anlage eines zentralvenösen Katheters

4. schriftliche Einwilligung des Patienten oder des gesetzlichen Betreuers für die Teilnahme in die Studie

5. V. jugularis interna und V. subclavia als Insertionsstelle

Die Ausschlusskriterien waren:

1. Alter unter 18 Jahren

2. Patienten bzw. Betreuer die nicht in der Lage waren, das Wesen der Studie zu begreifen und ihren Willen schriftlich zu bestimmen

3. V. femoralis als Insertionsstelle

\subsection{Studienablauf}

Initial wurden die ersten zehn Patienten (Testkohorte) nur von einem erfahrenen Studienarzt untersucht um die Prozedur allmählich zu erproben. Im Anschluss wurde bei noch 90 Patienten (Validierungskohorte) die Ultraschalluntersuchung durch verschiedene diensthabende Ärzte im ersten bis sechsten Weiterbildungsjahr durchgeführt. Zuvor erfolgte ein 30- bis 60-minütiges Training durch die Studienärzte für eine fokussierte Sonographie des Herzens durch einen subkostalen und apikalen Vierkammerblick mit einem Sektorschallkopf. Die Punkteure mussten mehr als 20 Katheter gelegt haben und wurden von einem erfahrenen Assistenzarzt oder Facharzt supervidiert.

Die Probanden erhielten ein Informationsblatt über das Wesen der Studie und die Aufbewahrung der personenbezogenen Daten. Nicht-einwilligungsfähige Patienten wurden nach Feststellung des mutmaßlichen Willens ebenfalls in die Studie eingeschlossen, da sich im intensivmedizinischen Bereich regelhaft die medizinisch gebotene Notwendigkeit der Anlage eines zentralvenösen Katheters ohne die Möglichkeit der schriftlichen Einwilligung durch den Patienten selbst oder dessen gesetzlichen 
Vertreter ergibt. Der mutmaßliche Willen des nicht-einwilligungsfähigen Patienten wurde vom Arzt aus einer eventuell vorliegenden Patientenverfügung oder durch das Gespräch mit den Angehörigen oder dem gesetzlichen Betreuer ermittelt und in einem speziellen Formular dokumentiert. Es wurde ausdrücklich formuliert, dass die Teilnahme an der Studie freiwillig ist und jederzeit widerrufen werden konnte (siehe Anhang). Nach Einholung der schriftlichen Einwilligung wurden die Patienten in die Studie eingeschlossen.

Die patientenbezogenen Merkmale Alter, Geschlecht, Größe, Gewicht, Blutdruck, Herzfrequenz und Beatmungstyp wurden dokumentiert. Der Patient wurde in Oberkörpertieflage (Trendelenburg-Position) von $10-15^{\circ}$ gelagert, um das Risiko einer Luftembolie zu vermindern und gleichzeitig einen besseren Füllungszustand der Venen zu erzielen. Das Arbeitsmaterial wurde unter sterilen Bedingungen vorbereitet und das Punktionsgebiet gründlich desinfiziert. Wir verwendeten drei-, vier- oder fünflumige, nicht-getunnelte Katheter (Triple-Lumen ARROWg+ard Blue mit Blue FlexTip $®$, Four- and Five-Lumen LOGICATH ${ }^{\mathrm{TM}}$ Kit, Fa. Teleflex Inc., Morrisville, North Carolina, USA).

Die V. jugularis oder die V. subclavia wurden entweder anhand von anatomischen Landmarken oder ultraschallgestützt aufgesucht. Zunächst erfolgte die Applikation des Lokalanästhetikums mit einer $21 \mathrm{G}$ oder $23 \mathrm{G}$ Nadel. Nach Ablauf der Einwirkzeit wurde das Gefäß mit einer $18 \mathrm{G}$ Kanüle in einem Winkel von 20-30 punktiert (Abb. 1).

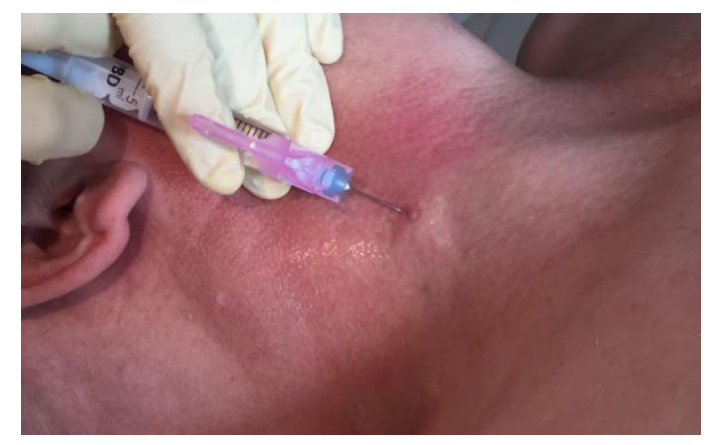

Abb. 1: Applikation der Lokalanästhesie.

Unter stetiger Aspiration bestätigte sich die intravenöse Lage der Kanüle durch das Einströmen von dunkelrotem Blut (Abb. 2).

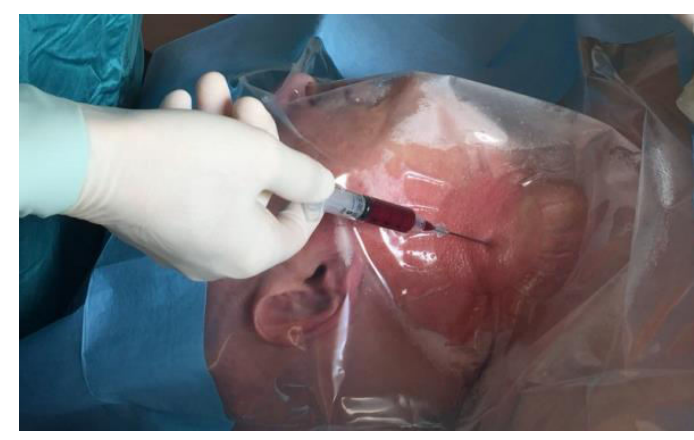

Abb. 2: Punktion des Gefäßes unter Aspiration. 
Anschließend wurde die Spritze von der Kanüle entfernt und der SeldingerFührungsdraht wurde ca. $10 \mathrm{~cm}$ vorgeschoben (Abb. 3).
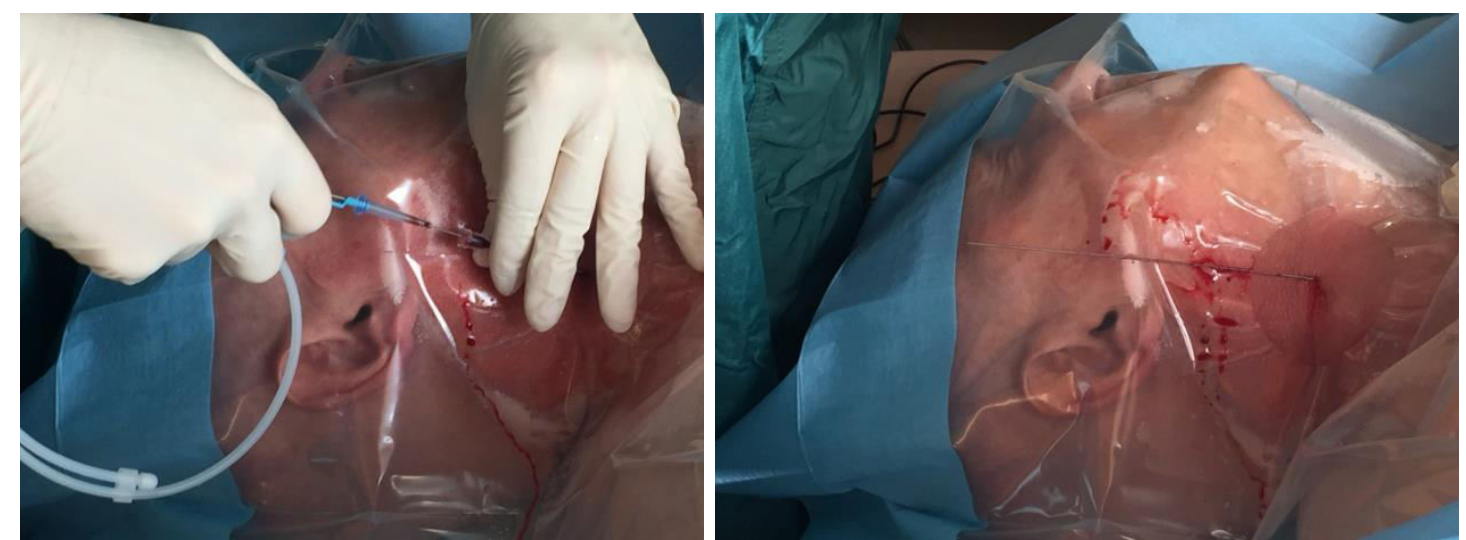

Abb. 3: Einführung des Seldinger-Führungsdrahtes.

Die Nadel wurde anschließend entfernt und mit Hilfe eines Skalpells und des entsprechenden Dilatators wurde die Einstichsstelle aufgeweitet (Abb. 4).
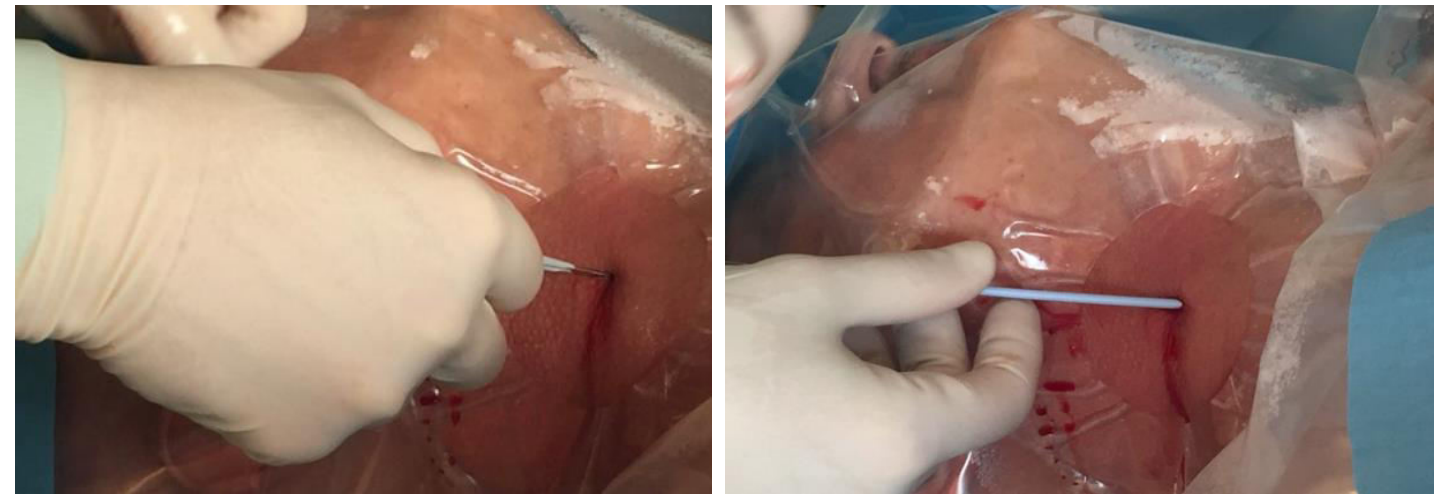

Abb. 4: Dilatation des Stichkanals.

Der Katheter wurde über den Draht eingeführt. Nach Entfernung des Führungsdrahtes erfolgte die Aspirationskontrolle, und alle Schenkel wurden mittels Kochsalzlösung $(0,9 \%)$ gespült (Abb. 5). Der Katheter wurde anschließend mit Nahtmaterial fixiert und mit einem sterilen Pflaster abgedeckt.
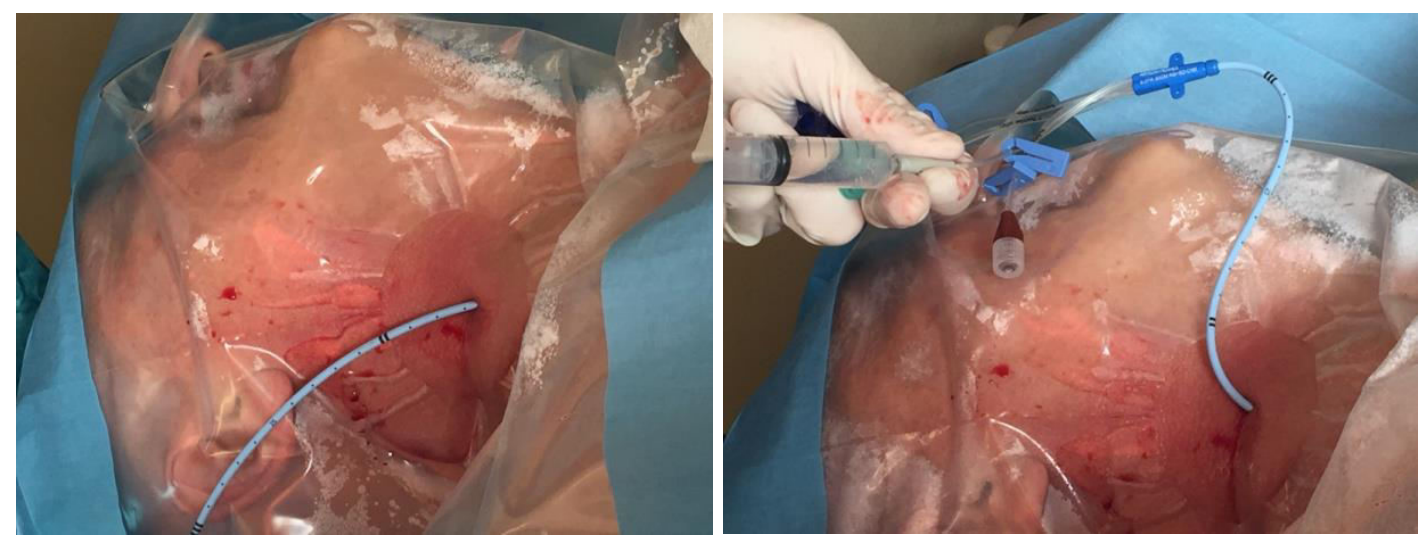

Abb. 5: Entfernung des Führungsdrahtes und Einführung des Katheters. Aspirationskontrolle und Spülung mit 0,9\%iger Kochsalzlösung. 
Unmittelbar nach der Anlage des zentralvenösen Katheters wurde die MTRA der Radiologie verständigt und die Uhrzeit dokumentiert. Anschließend wurde die BMode (brightness modulation) Ultraschalluntersuchung mit einem Sektorschallkopf von subxiphoidal oder apikal je nach anatomischen Gegebenheiten durchgeführt. Wir verwendeten entweder das Esaote MyLab5 ${ }^{\mathrm{TM}}$ oder das Esaote MyLabGold ${ }^{\mathrm{TM}}$ Ultraschallgerät (Fa. Esaote S.p.A., Genua, Italien) (Abb. 6).

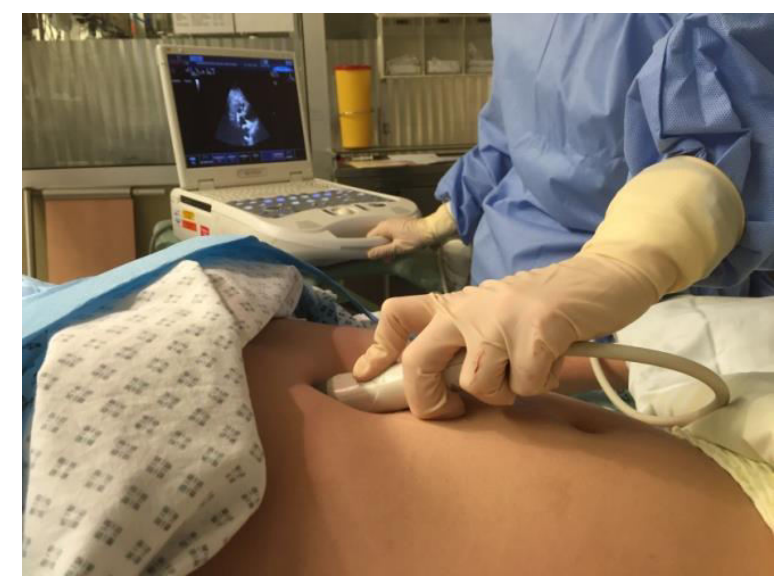

Abb. 6: Durchführung der fokussierten Echokardiographie.

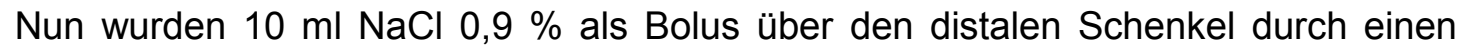
zweiten Arzt appliziert. Die Darstellung des Flüssigkeitsbolus (flush) im rechten Vorhof wurde nach Vezzani et al. (2010) als sofort vorhanden (auch: rapid atrial swirl sign, RASS [weniger als zwei Sekunden nach Injektion], verspätet (mehr als zwei Sekunden nach Injektion) oder abwesend beurteilt (Abb. 7). Nur das sofort sichtbare RASS wurde als korrekte Positionierung gewertet. Die Lage wurde als inkorrekt definiert wenn die Spitze zu tief im rechten Atrium oder nach kranial disloziert war und eine Korrektur durch den Punkteur notwendig war.

Die Untersuchung konnte bis zu dreimal durchgeführt werden und, wenn im flushTest eine Fehllage vermutet wurde, erfolgte die Korrektur der Katheterlage.

Die Ergebnisse der Ultraschall-Untersuchungen wurden in Video-Form (sog. "loop“) auf der Festplatte des Ultraschallgerätes gespeichert und zu Analysezwecken auf ein externes Speichermedium exportiert. Die Dauer der Untersuchung und der durchführende Arzt wurden dokumentiert. Eine Stichprobe mit $30 \%$ aller Videos wurde zufällig ausgewählt und von zwei unabhängigen Bewertern evaluiert. 


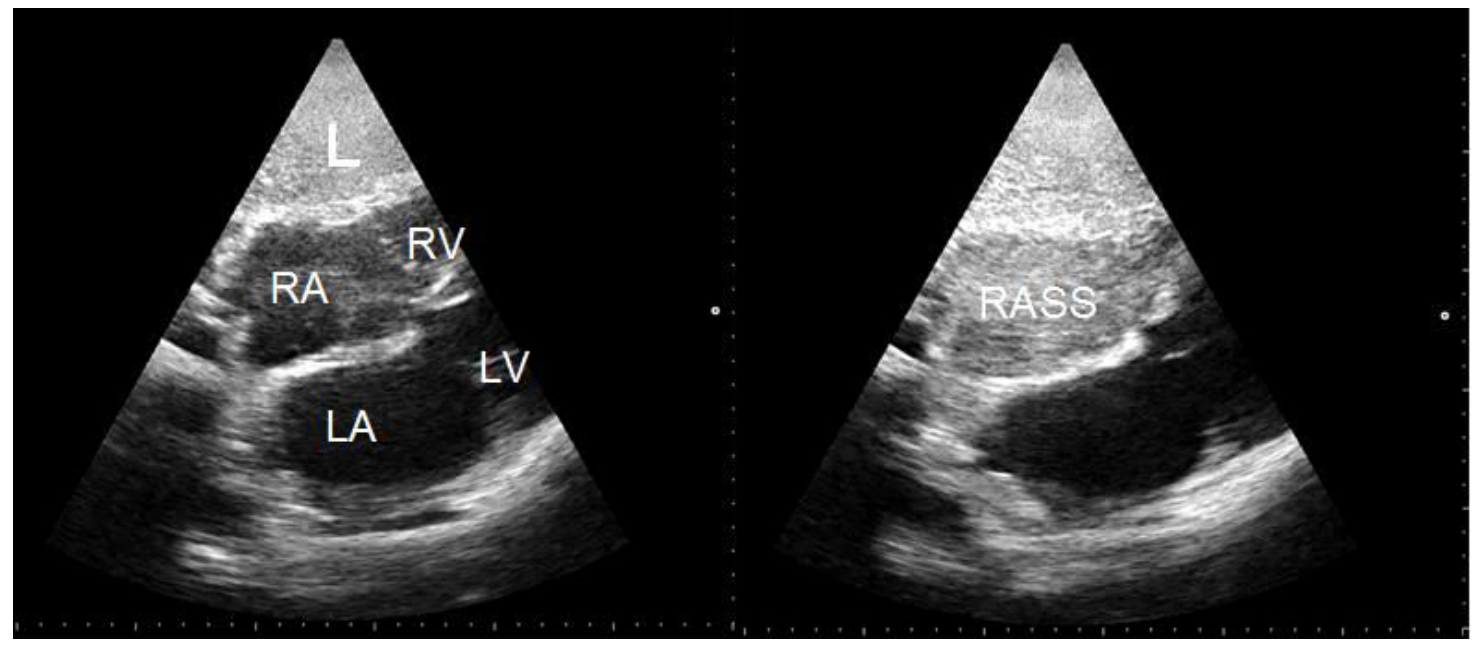

Abb. 7: Demonstration des RASS in subkostalem Vierkammerblick.

Verwendete Abkürzungen: RA: rechtes Atrium, LA: linkes Atrium, RV: rechter Ventrikel, LV: linken Ventrikel, L: Leber, RASS: Rapid Atrial Swirl Sign.

Das Röntgen des Thorax wurde in a.p.-Projektion im Liegen durchgeführt und die Bilder wurden in digitaler Form im PACS (Centricity Enterprise Web V 3.0, 2006 GE Medical Systems) gespeichert. Durch Stoppen der Zeit wurde der Zeitbedarf ermittelt, bis das Bild im PACS abgerufen werden konnte.

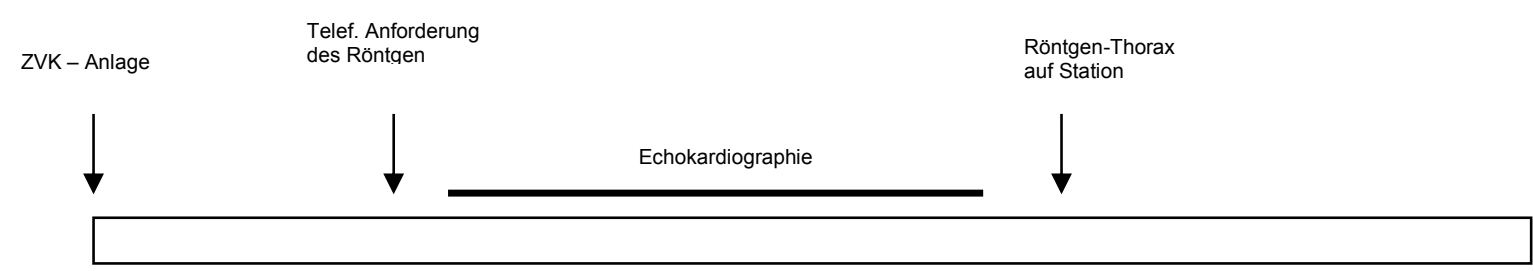

Abb. 8: Zeitlicher Ablauf der Studie.

Verwendete Abkürzungen: ZVK: zentraler Venenkatheter.

Bei der Befundung des Röntgen-Bildes konzentrierten wir uns in den Strukturen des Mediastinums (Abb. 9). In unserer Studie wurde die Carina tracheae als Orientierungsmarke verwendet, daraufhin wurde die Trachea mit der Bifurkation in die Hauptbronchien aufgesucht und eine Parallele durch die Carina gezeichnet, um dies zu verdeutlichen (Abb. 10). Dann erfolgte gemäß der Einteilung der Röntgen-Zonen von Weekes et al. (2014) sowie Fletcher und Bodenham (2000) die modifizierte Unterteilung der möglichen radiologischen ZVK-Positionen in vier Zonen (Abb. 11) (Fletcher und Bodenham 2000; Weekes et al. 2014).

- Zone A entsprach der Region kranial der Carina und repräsentierte den proximalen Bereich der Vena cava superior

- Zone B entsprach der Region kaudal der Carina und repräsentierte den distalen Bereich der Vena cava superior

- Zone C entsprach der Vena brachiocephalica auf der linksseitigen Hälfte des Mediastinums

- Zone D entsprach dem rechten Vorhof 
Katheterspitzen in den Zonen A und B wurden als korrekt liegend und in den Zonen $C$ und $D$ als falsch liegend bewertet. Die Auswertung der Bilder erfolgte durch einen unabhängigen Untersucher und einen verblindeten Radiologen.

Nach Beendigung der Untersuchungen wurden alle Ergebnisse im Untersuchungsprotokoll (siehe Anhang) erfasst. Die Fotodokumentation wurde mit Einwilligung des Patienten durchgeführt (siehe Anhang).

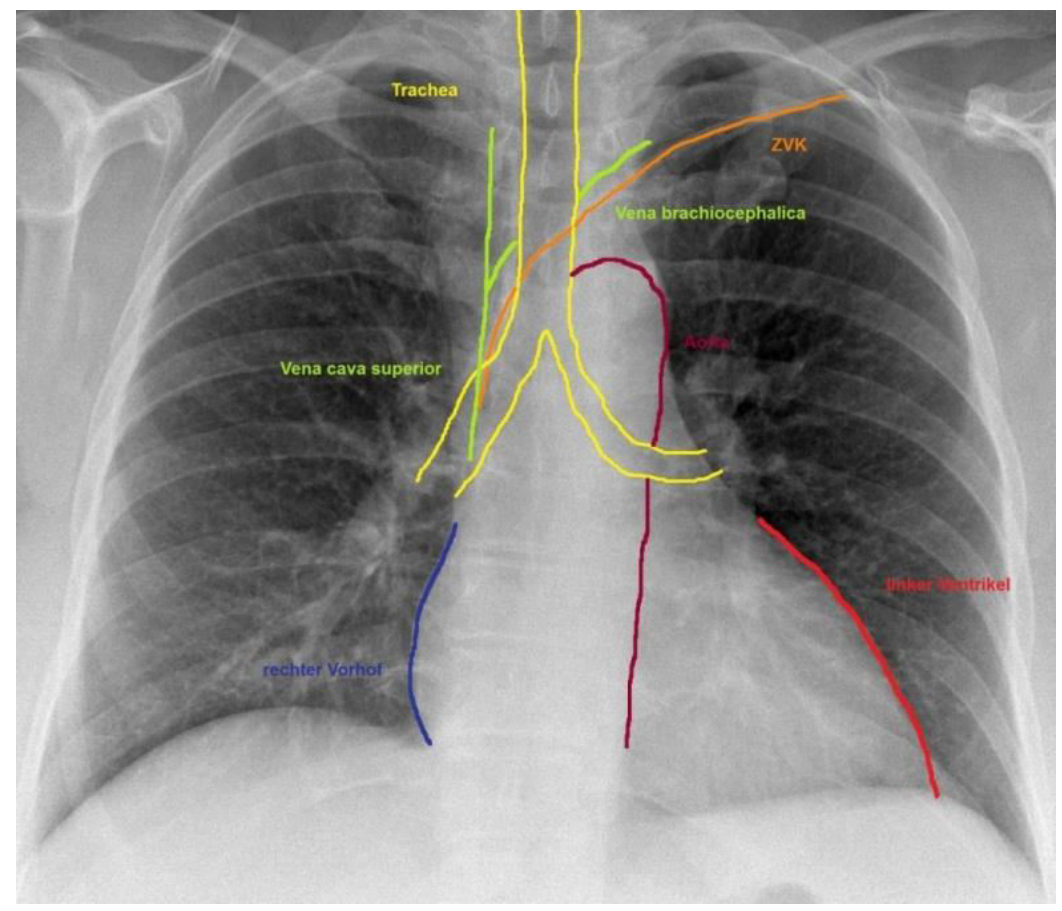

Abb. 9: Posterior - anterior - Röntgenaufnahme des Thorax nach ZVK-Anlage. Die wichtigsten Strukturen wurden farblich gekennzeichnet. Man erkennt den über die linke V. subclavia eingebrachten ZVK, dessen Spitze kurz unterhalb der Carina liegt.

Verwendete Abkürzungen: ZVK: zentraler Venenkatheter.

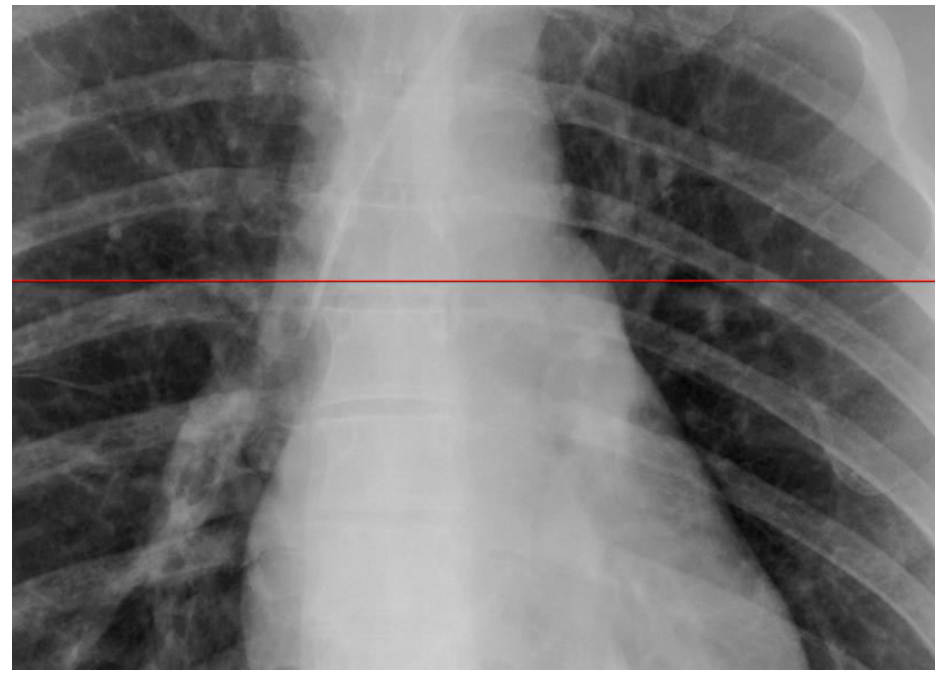

Abb. 10: Posterior - anterior - Röntgenaufnahme des Thorax nach ZVK-Anlage mit gezeichneter Linie durch die Carina. 


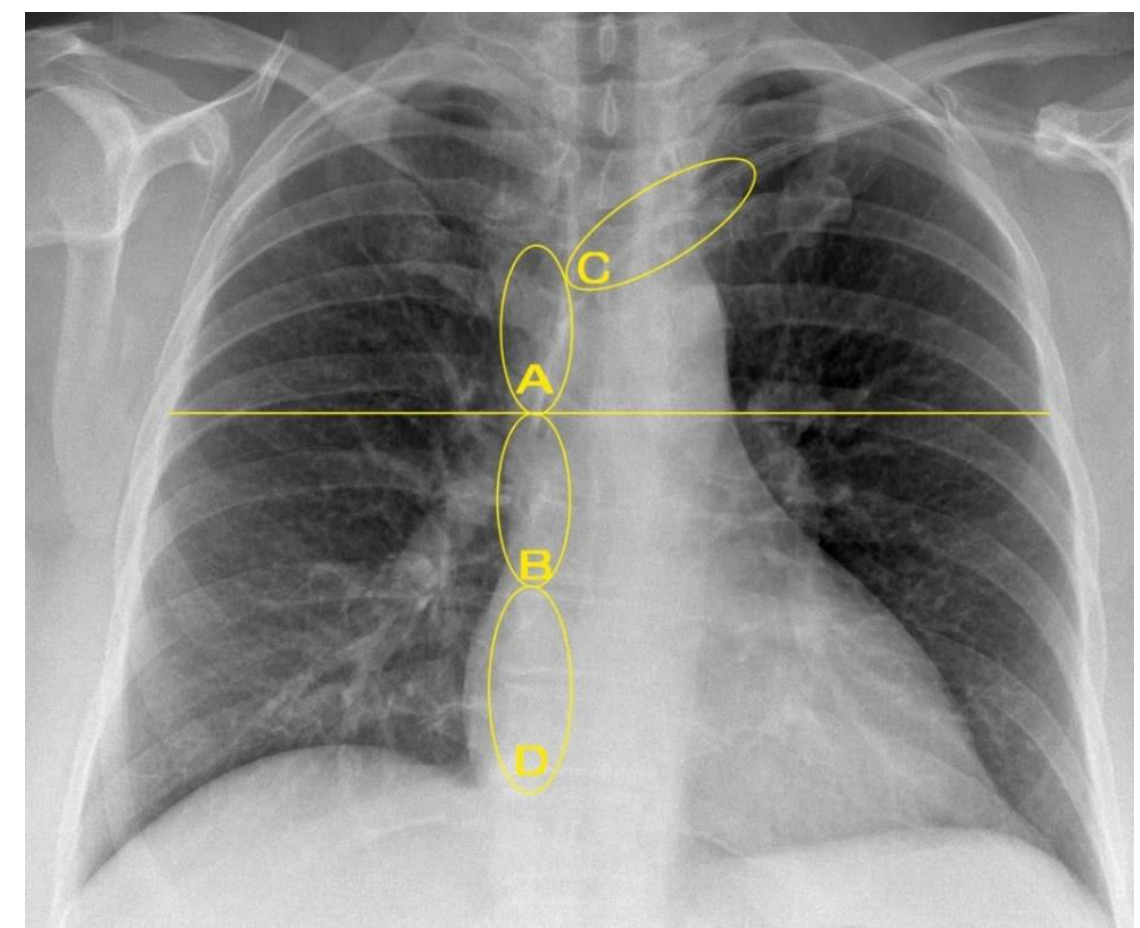

Abb. 11: Unterteilung der möglichen ZVK - Positionen in vier Zonen. Zone A entspricht den proximalen Bereich der V. cava superior, Zone B den distalen Bereich der V. cava superior, Zone C die V. brachiocephalica und Zone D den rechten Vorhof. Katheterspitzen in den Zonen A und B wurden als korrekt gelegt bezeichnet.

\subsection{Statistische Auswertung}

Für die statistische Auswertung erfolgte eine Beratung durch Mitarbeiter des Instituts für Medizinische Statistik der Georg-August-Universität Göttingen. Die erhobenen Daten wurden mit Hilfe der Programme Microsoft Excel und Word (Microsoft Corporation, Redmond, Washington, USA) sortiert und die deskriptive Statistik sowie die Testverfahren wurden mit der Statistik-Software GraphPad Prism version 5.00 (GraphPad Software, San Diego, Kalifornien, USA) sowie MedCalc Statistical Software Version 16.4.3 (MedCalc Software bvba, Ostende, Belgien) analysiert.

Die Prüfung auf Normalverteilung erfolgte mit dem Shapiro-Wilk-Test. Für die normalverteilten Stichproben wurde der t-Test angewendet und für verteilungsfreie Stichproben der Wilcoxon- oder Mann-Whitney U-Test. Die Messungen beim gleichen Patienten wurden mit dem Wilcoxon signed rank-Test analysiert. Um die Echokardiographie mit dem Röntgen-Thorax zu vergleichen, wurden die Sensitivität, Spezifität sowie der positive und negative Vorhersagewert und die Likelihood Ratios berechnet. Als Gold-Standard für die korrekte Lage des Katheters wurde das RöntgenThorax verwendet. Für die Zuverlässigkeit der Röntgen-Befundung zwischen den zwei unabhängigen Bewertern wurden der Korrelationskoeffizient nach Pearson und die Methode nach Bland-Altman (Referenz) eingesetzt. Die Übereinstimmung der Evaluation der fokussierten Echokardiographie zwischen zwei Ratern wurde mit dem Kappa-Koeffizient nach Cohen berechnet. Der Weiteren wurden die PearsonKorrelation zwischen dem BMI und der Länge der Echokardiographie analysiert. Ein signifikanter Unterschied zwischen den Testergebnissen wurde mit einem Signifikanzniveau des $p$-Wertes von 0,05 (beziehungsweise $5 \%$ ) angenommen. 


\section{$3 \quad$ Ergebnisse}

\subsection{Studienkohorten}

Von November 2014 bis April 2016 wurden insgesamt 100 Patienten in unsere prospektive, randomisierte Studie eingeschlossen. Um den primären und sekundären Endpunkt besser zu analysieren wurden die Patienten in zwei Kohorten eingeteilt: eine Testkohorte und eine Validierungskohorte. Als Testkohorte wurden die ersten zehn Patienten herangezogen, die vom erfahrenen Studienarzt untersucht wurden. Die restlichen 90 Patienten (Validierungskohorte) wurden von den diensthabenden Ärzten der Intermediate Care (IMC-Station 1021) und Intensivstation (ICU-Station 1022) der Klinik für Nephrologie und Rheumatologie untersucht.

In der Testkohorte waren fünf weibliche und fünf männliche Patienten, das Durchschnittsalter betrug 70 Jahre. Der jüngste Patient 19 und der älteste 91 Jahre alt. In der Validierungskohorte waren 47 weibliche und 43 männliche Patienten mit einem mittleren Alter von 68 Jahren. Die Mehrheit der Patienten der Testkohorte (80 \%) und der Validierungskohorte (89\%) waren spontanatmend, die zentralvenösen Katheter hatten meistens drei Lumen (Testkohorte $90 \%$, Validierungskohorte $96 \%$ ) und die rechte $V$. jugularis interna war das am häufigsten punktierte Gefäß (Testkohorte 50 $\%$, Validierungskohorte $53,3 \%$ ) im Vergleich zur linken $\mathrm{V}$. jugularis interna (Testkohorte $40 \%$, Validierungskohorte 41,1\%). Die Punktion wurde in $70 \%$ der Fälle der Testkohorte und $82,2 \%$ der Validierungskohorte mit Hilfe einer sonographischen Darstellung des Gefäßes durchgeführt. Die V. subclavia wurde nur in seltenen Fällen mit Hilfe der landmark-Technik oder sonographisch gesteuert verwendet. Die oben genannten Charakteristika zeigten keinen signifikanten Unterschied zwischen beiden Kohorten (Tab. 5). Ein Patient mit einem BMl von $40 \mathrm{~kg} / \mathrm{m}^{2}$ wurde wegen nicht durchführbarer Echokardiographie aus der Studie ausgeschlossen.

Tab. 5: Charakteristika der Studienkohorten.

Verwendete Abkürzungen: min: minimum, max: maximum, IMC: Intermediate Care, ICU: Intensive Care Unit, NIV: non-invasive ventilation, IV: invasive ventilation, ZVK: zentraler Venenkatheter, R: rechte, L: linke, V.: Vena, US: Ultraschall.

\begin{tabular}{|l|l|l|}
\hline & Testkohorte (n=10) & $\begin{array}{l}\text { Validierungskohorte } \\
(\mathbf{n = 9 0 )}\end{array}$ \\
\hline $\begin{array}{l}\text { Alter (Median, min }- \\
\text { max) }\end{array}$ & $70,0(36-91)$ & $68,0(19-90)$ \\
\hline $\begin{array}{l}\text { Geschlecht } \\
\text { (männlich / weiblich) }\end{array}$ & $5 / 5$ & $43 / 47$ \\
\hline Station (IMC / ICU) & $5 / 5$ & $52 / 38$ \\
\hline $\begin{array}{l}\text { Beatmungstyp } \\
\text { Keine }\end{array}$ & $8(80 \%)$ & $80(89 \%)$ \\
NIV & $1(10 \%)$ & $5(5,5 \%)$ \\
IV & $1(10 \%)$ & $5(5,5 \%)$ \\
\hline ZVK-Typ & $9(90 \%)$ & $86(96 \%)$ \\
3-Lumen & $1(10 \%)$ & $3(3 \%)$ \\
4-Lumen & $0(0 \%)$ & $1(1 \%)$ \\
5-Lumen & $5(50 \%)$ & $48(53,3 \%)$ \\
\hline $\begin{array}{l}\text { Punktionsort } \\
\text { R V. jugularis interna }\end{array}$ & $5(50 \%)$ & \\
\hline
\end{tabular}




\begin{tabular}{|l|l|l|}
\hline L V. jugularis interna & $4(40 \%)$ & $37(41,1 \%)$ \\
R V. subclavia & $0(0 \%)$ & $2(2,2 \%)$ \\
L V. subclavia & $1(10 \%)$ & $3(3,3 \%)$ \\
\hline $\begin{array}{l}\text { US-gesteurte Punk- } \\
\text { tion (nein / ja) }\end{array}$ & $3 / 7(30 \% / 70 \%)$ & $16 / 74(17,8 \% / 82,2 \%)$ \\
\hline
\end{tabular}

\subsection{Analyse der postprozeduralen radiologischen Kontrolle}

Die postprozedurale radiologische Kontrolle des zentralvenösen Katheters erfolgte durch einen Radiologen und die diensthabenden Ärzte der jeweiligen Station. Alle Röntgenaufnahmen wurden von einem unabhängigen Untersucher und einem Radiologen interpretiert, die Position der Katheterspitze entsprechend der zuvor definierten vier Zonen dokumentiert (Abb. 11). Insgesamt $74 \%$ der Katheter lagen korrekt in den Zonen A und B, 3 \% lagen zu hoch in der Zone C und $23 \%$ zu tief im rechten Vorhof (Abb. 12). Die Entscheidung einer Korrektur wurde ausschließlich vom Punkteur getroffen. In einem Fall zeigte sich die Katheterspitze in Projektion auf die Aorta thorakalis descendens. In der folgenden CT-Untersuchung des Thorax hat sich die Diagnose einer persistierenden linken $\mathrm{V}$. cava superior herausgestellt. Sie ist die häufigste Fehlbildung des thorakalen venösen Systems mit einer Inzidenz von 0,5\% in der Bevölkerung und resultiert aus einer fehlenden Obliteration der V. cardinalis sinistra. Diese Fehlbildung führt zu einer abnormen elektrophysiologischen Funktion des Herzens, die zu Herzrhythmusstörungen führen kann (Morgan et al. 2015). Wegen der erhöhten Gefahr für das Auftreten von Arrhythmien wurde der Katheter sofort entfernt.

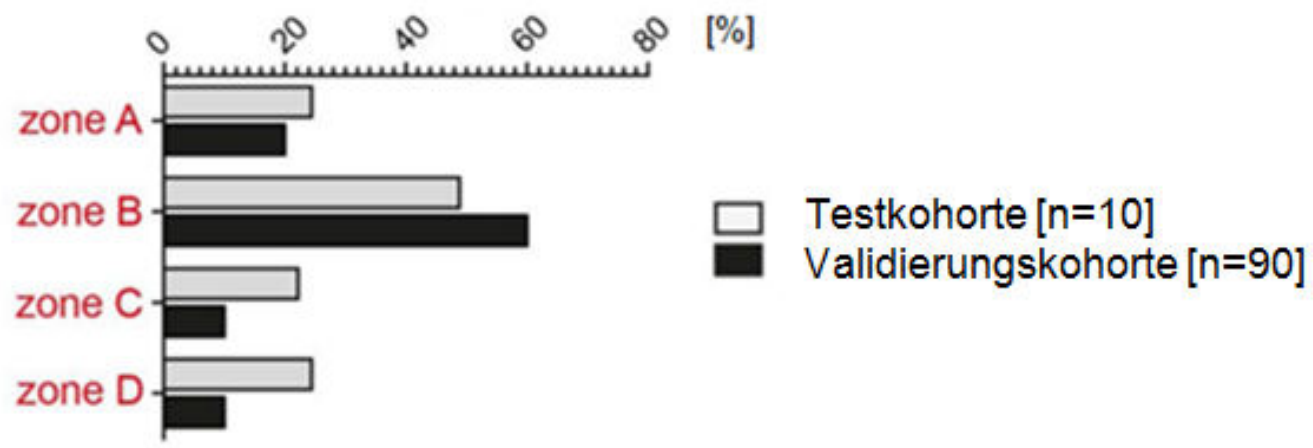

Abb. 12: Verteilung der Katheter-Lage in den vier radiologischen Zonen.

Die Bewertung der Röntgenaufnahmen durch zwei unabhängige Untersucher zeigte mit Hilfe der logistischen Regression eine positive Korrelation $\left(r^{2}=0.8665\right)$. In der Bland-Altman-Methode betrug die mittlere Differenz zwischen beiden Messungen $0.09 \mathrm{~mm}$, die Übereinstimmung der Beurteilung der Höhe der Katheterspitze zwischen beiden Untersuchern war somit sehr gut (Abb. 13). 
A

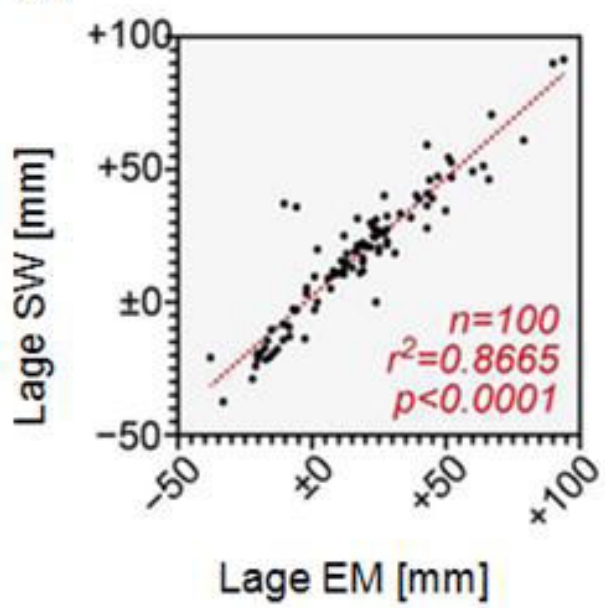

B

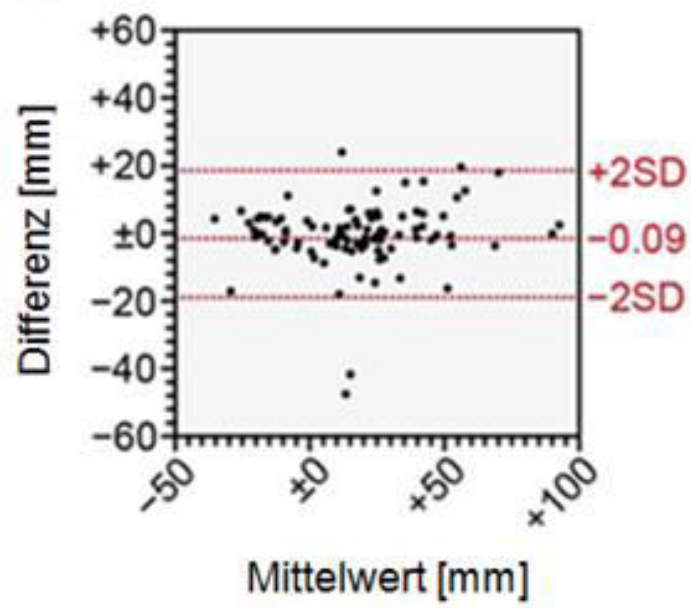

Abb. 13: (A) Korrelation der Bewertung der Röntgenaufnahmen zwischen zwei unabhängigen Untersuchern mit linearer Regression. (B) Bland-Altman-Plot mit Darstellung der Differenz zum Mittelwert der Katheterposition in Relation zur Carina durch zwei Untersucher.

Verwendete Abkürzungen: $\mathrm{EM}=$ Eirini Mavropoulou, $\mathrm{SW}=$ Susanne Wienbeck, mm: Millimeter.

\subsection{Das echokardiographische rapid atrial swirl sign - RASS}

Für die fokussierte Echokardiographie wurden zwei Anlotungen genutzt: der subkostale und der apikale Vierkammerblick. Die subkostale Schnittebene wurde bei der Mehrzahl der Patienten (86\%) angewendet, während der apikale Vierkammerblick nur bei 15 Patienten aufgrund der anatomischen Gegebenheiten benötigt wurde. Im Gesamtkollektiv konnten mit Hilfe der Röntgenaufnahmen zwölf Fehllagen detektiert werden. Ein positiver flush-Test (weniger als zwei Sekunden nach Injektion) wurde bei 83 von 100 Patienten beschrieben; dies wurde als negativer screening-Test interpretiert. Ein verspäteter flush wurde bei neun Patienten beobachtet, bei acht Patienten konnte kein Flüssigkeitsbolus dargestellt werden (Tab. 6). Ein verspäteter oder abwesender flush wurde als positiver screening-Test gewertet.

Tab. 6: Ergebnisse der Röntgenaufnahmen und der Echokardiographie in beiden Kohorten.

\begin{tabular}{|l|l|l|l|}
\hline & $\begin{array}{l}\text { Testkohorte } \\
(\mathbf{n = 1 0 )}\end{array}$ & $\begin{array}{l}\text { Validierungskohorte } \\
(\mathbf{n = 9 0 )}\end{array}$ & $\begin{array}{l}\text { Gesamtkollektiv } \\
(\mathbf{n = 1 0 0 )}\end{array}$ \\
\hline $\begin{array}{l}\text { CXR korrekte Lage } \\
\text { (nein/ja) }\end{array}$ & $2 / 8$ & $10 / 80$ & $12 / 88$ \\
\hline $\begin{array}{l}\text { Echokardiographie } \\
\text { flush sofort }\end{array}$ & 7 & 76 & 83 \\
flush verspätet & 1 & 8 & 9 \\
flush abwesend & 2 & 6 & 8 \\
\hline Anlotung & 4 & $11^{*}$ & $15^{*}$ \\
Apikal & 6 & 80 & 86 \\
Subcostal & 6 & & \\
\hline
\end{tabular}

${ }^{*}$ Bei einem Patienten wurden beide Anlotungen angewendet. Verwendete Abkürzungen: CXR: chest $X$-ray. 


\subsection{Testcharakteristika des rapid atrial swirl signs - RASS}

Die Sensitivität der fokussierten Echokardiographie im Vergleich zum Röntgen lag bei $100 \%$ in beiden Kohorten, was indiziert, dass im Falle eines fehlenden RASS die Katheterlage auch auch radiologisch nicht als korrekt eingestuft wird. Die Spezifität betrug $87,5 \%$ in der Testkohorte und $95 \%$ in der Validierungskohorte. Der positiv prädiktive Wert für eine falsche radiologische Lage mit negativem RASS betrug $66,7 \%$ in der Testkohorte und $71,4 \%$ in der Validierungskohorte. Der negative prädiktive Wert war $100 \%$ in beiden Kohorten (Tab. 7). Die Likelihood Ratio für positive Testresultate lag bei 17,6 und für negative Resultate bei 0,00, was eine hohe diagnostische Aussagekraft der Echokardiographie bedeutet.

Tab. 7: Testcharakteristika des rapid atrial swirl sigs in der Test- und Validierungskohorte.

\begin{tabular}{|c|c|c|c|c|}
\hline & $\begin{array}{l}\text { CXR Lage falsch } \\
\text { (Test / Validierung) } \\
\text { [Gesamt] }\end{array}$ & $\begin{array}{l}\text { CXR Lage korrekt } \\
\text { (Test / Validierung) } \\
\text { [Gesamt] }\end{array}$ & Gesamt & $\begin{array}{l}\text { Testcharakteristika } \\
\text { (Gesamt) }\end{array}$ \\
\hline $\begin{array}{l}\text { Screening- } \\
\text { Test positiv, } \\
\text { RASS } \\
\text { negativ }\end{array}$ & $2 / 10$ [12] & $1 / 4[5]$ & 17 & \multirow{3}{*}{$\begin{array}{l}\text { Sensitivität: } 100 \% \\
(95 \% \text { KI 73,54-100 \%) } \\
\text { Spezifität: 94,32\% } \\
(95 \% \text { KI 87,24-98,13\%) } \\
\text { LR+: 17,6 } \\
(95 \% \text { KI 7,51-41,23) } \\
\text { LR-: 0,00 } \\
\text { PPV: 70,59 \% } \\
(95 \% \text { KI 44,04-89,69 \%) } \\
\text { NPV: } 100 \% \\
(95 \% \text { KI 95,65-100\%) }\end{array}$} \\
\hline \begin{tabular}{l}
\multicolumn{2}{l}{ Screening- } \\
Test nega- \\
tiv, \\
RASS posi- \\
tiv
\end{tabular} & 0 / 0 [0] & 7 / 76 [83] & 83 & \\
\hline Gesamt & 12 & 88 & 100 & \\
\hline
\end{tabular}

Verwendete Abkürzungen: CXR: chest X-ray, RASS: rapid atrial swirl sign, KI: Konfidenzintervall, LR: Likelihood Ratio, PPV: Positive Predictive Value, NPV: Negative Predictive Value.

\subsection{Die interrater-Reliabilität des rapid atrial swirl signs - RASS}

Um die interrater-Reliabilität des rapid atrial swirl signs zu prüfen, wurden $30 \%$ aller Videoaufzeichnungen von zwei unabhängigen Untersuchern beurteilt. Die drei möglichen Variablen waren der positive, ein verspäteter und ein nicht vorhandener flush. Für die Bewertung der Übereinstimmung zwischen beiden Untersuchern wurde der Cohens-Kappa-Koeffizient berechnet. Dieser betrug 0,726 (Standardfehler 0,121, 95\% Konfidenzintervall 0,488 - 0,964) und zeigte somit eine gute Übereinstimmung zwischen den Bewertern an (Tab. 8). 
Tab. 8: Die interrater-Reliabilität bei der Bewertung des rapid atrial swirl signs mit drei Variablen.

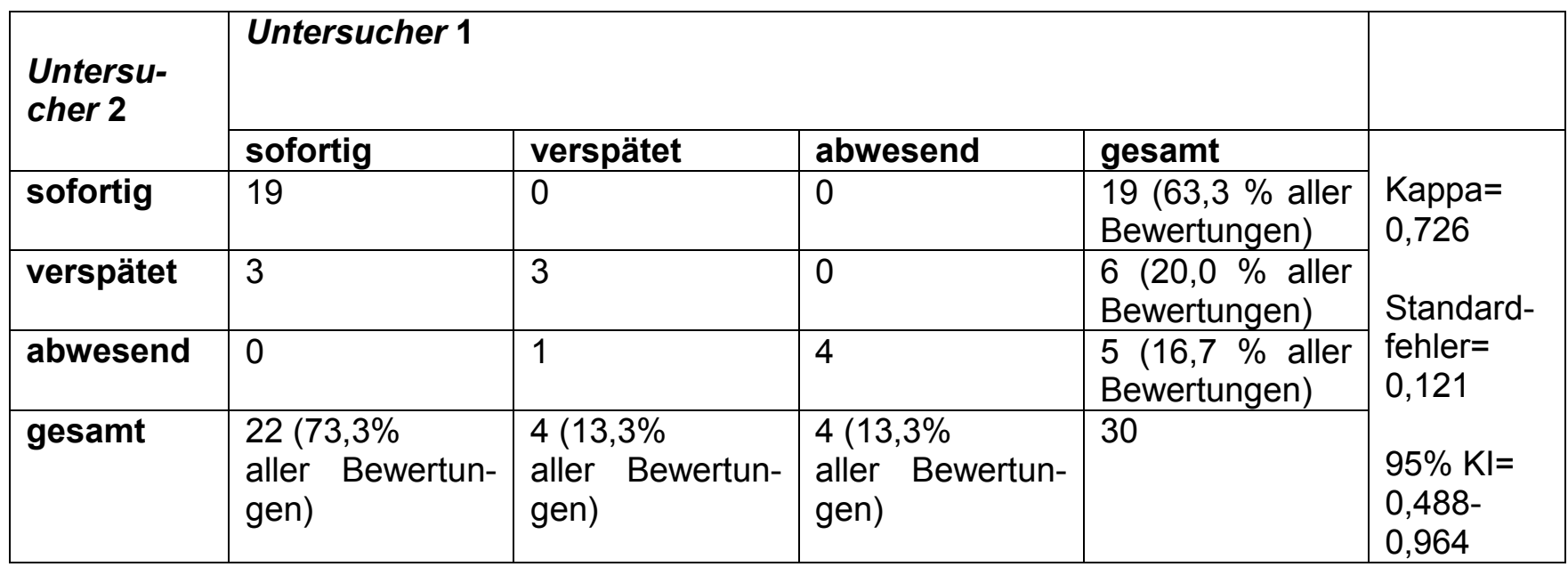

Verwendete Abkürzungen: KI: Konfidenzintervall.

Die möglichen Variablen wurden im zweiten Schritt dichotomisiert, d. h. ein sofortiger flush wurde als negativer screening-Test für eine Fehllage bewertet und ein verspäteter oder abwesender flush als positives. Hier zeigte sich eine etwas härtere interrater-Reliabilität mit einem Cohens-Kappa-Koeffizienten von 0,772 (Standardfehler 0,122, $95 \%$ Konfidenzintervall KI 0,533 - 1.0, Tab. 9).

Tab. 9: Die Interrater-Reliabilität bei der Bewertung des rapid atrial swirl signs mit zwei Variablen.

\begin{tabular}{|c|c|c|c|c|}
\hline \multirow{2}{*}{$\begin{array}{l}\text { Untersucher } \\
2\end{array}$} & \multicolumn{3}{|l|}{ Untersucher 1} & \\
\hline & positiv & negativ & gesamt & \multirow{4}{*}{$\begin{array}{l}\text { Kappa=0,772 } \\
\text { Standardfehler= } \\
0,122 \\
95 \% \mathrm{Kl}=0,533-1,0\end{array}$} \\
\hline positiv & 19 & 0 & $\begin{array}{l}19(63,3 \% \text { aller } \\
\text { Bewertungen) }\end{array}$ & \\
\hline negativ & 3 & 8 & $\begin{array}{l}11 \text { (36,7\% aller } \\
\text { Bewertungen) }\end{array}$ & \\
\hline gesamt & $\begin{array}{l}22(73,3 \% \text { aller } \\
\text { Bewertungen) }\end{array}$ & $\begin{array}{l}8 \quad(26,7 \% \text { aller } \\
\text { Bewertungen) }\end{array}$ & 30 & \\
\hline
\end{tabular}

Verwendete Abkürzungen: KI: Konfidenzintervall.

\subsection{Vergleich zwischen Echokardiographie und Röntgen}

Nach der Anlage des zentralvenösen Katheters wurde die MTRA der Radiologie telefonisch verständigt. Die durchschnittliche Zeit, bis das Röntgenbild im PACS abrufbar war, belief sich auf 59,5 Minuten (21 - 130) für die Testkohorte und auf 48,5 Minuten (13 - 254) für die Validierungskohorte. Es ließ sich kein signifikanter Unterschied zwischen beiden Gruppen feststellen $(p=0,5162)$. Die mittlere Dauer für die Durchführung und Auswertung der fokussierten Echokardiographie betrug fünf Minuten (2 11 Minuten in der Testkohorte und $1-28$ Minuten in der Validierungskohorte) in beiden Gruppen $(p=0,4666)$, was einen erheblichen Vorteil gegenüber dem Röntgen bedeutet (Abb. 14). Die durchschnittliche Dauer für die Echokardiographie war mit fünf Minuten jeweils signifikant geringer als für das Röntgen mit 50 Minuten in beiden Gruppen (Testkohorte $p=0,011$ und Validierungskohorte $p<0,001$ mit dem MannWhitney U-Test). 


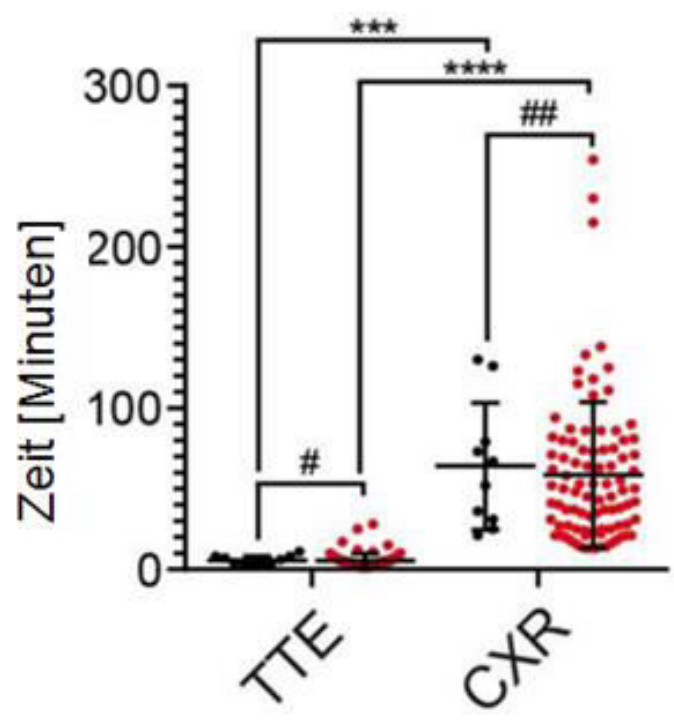

Testkohorte [n=10]

Validierungskohorte $[\mathrm{n}=90]$

\begin{abstract}
Abb. 14: Die zeitliche Dauer für die Echokardiographie und das Röntgen in der Test- und Validierungskohorte. Die durchschnittliche Dauer für die Echokardiographie zeigt sich mit Hilfe des Wilcoxon-Vorzeichen-Rang-Tests mit 5 Minuten signifikant kurzer als für das Röntgen (49,5 Minuten). Verwendete Symbole und Abkürzungen: TTE: transthorakale Echokardiographie, CXR: chest $X$-ray, $\# p=0,4666, \# \# p=0,5162,{ }^{* * *} p=0,002,{ }^{* * * *} p<0,0001$.
\end{abstract}

\title{
3.7 Der Ausbildungsgrad der Assistenzärzte
}

In unserer prospektiven Studie haben insgesamt 22 Assistenzärzte der Intermediate Care (IMC-Station 1021) und Intensivstation (ICU-Station 1022) teilgenommen, die nach einer 30- bis 60-minütiger Einweisung durch die Studienärzte die fokussierte Echokardiographie durchgeführt und befundet haben. Sie wurden anhand des Weiterbildungsjahres drei Gruppen zugeteilt. In der ersten Gruppe waren elf Assistenzärzte aus dem ersten und zweiten Weiterbildungsjahr mit einem Durchschnitt von 2,45 Echokardiographien pro Arzt. Die fünf Ärzte der zweiten Gruppe hatten eine höhere mittlere Untersuchungsanzahl von acht. Die sechs Ärzte der dritten Gruppe untersuchten durchschnittlich 5,5 Patienten (Tab. 10).

Tab. 10: Zusammensetzung der Gruppen und mittlere Anzahl von Untersuchungen pro Assistenzarzt.

\begin{tabular}{|l|l|l|l|}
\hline & $\begin{array}{l}\text { Weiterbildungsjahr } \\
\mathbf{1 - 2}\end{array}$ & $\begin{array}{l}\text { Weiterbildungsjahr } \\
\mathbf{3 - 4}\end{array}$ & $\begin{array}{l}\text { Weiterbildungsjahr } \\
\mathbf{5}-\mathbf{6}\end{array}$ \\
\hline $\begin{array}{l}\text { Anzahl der Assis- } \\
\text { tenzärzte }\end{array}$ & 11 & 5 & 6 \\
\hline $\begin{array}{l}\text { mittlere Anzahl von } \\
\text { Tests pro Arzt }\end{array}$ & 2,45 & 8 & 5,5 \\
\hline
\end{tabular}

\subsection{Testcharakteristika des RASS in den verschiedenen Gruppen der Assistenzärzte}

Die Assistenzärzte der zweiten und dritten Gruppe führten mehr Untersuchungen als die Ärzte im ersten und zweiten Weiterbildungsjahr durch (40 und 33 gegenüber 27). Die Testcharakteristika zeigten sich jedoch ähnlich in allen Gruppen. Die erfahrenen 
Weiterbilungsassistenten im fünften und sechsten Weiterbildungsjahr zeigten den höchsten positiven prädiktiven Wert von $75 \%$ und die höchste Likelihood Ratio mit 34. Trotzdem wird in den Ergebnissen abgebildet, dass die fokussierte Echokardiographie auch nach einem kurzen Training einfach und sicher durchgeführt werden kann. Die ermittelte Sensitivität beträgt überall $100 \%$, also ein positiver RASS (negativer screening-Test für eine Fehllage) kann eine korrekte Lage des Katheters bestätigen. Die Spezifität variiert in allen Gruppen zwischen $91,43 \%$ und 97,06 \%, sodass bei verspätetem oder abwesendem flush (negativer RASS oder positiver screeningTest) eine potentielle Fehllage des Katheters vorhergesagt werden kann (Tab. 11).

Tab. 11: Testcharakteristika zwischen den verschiedenen Gruppen der Assistenzärzte.

\begin{tabular}{|c|c|c|c|}
\hline & $\begin{array}{l}\text { Weiterbildungsjahr } \\
1-2 \\
(n=27)\end{array}$ & $\begin{array}{l}\text { Weiterbildungsjahr } \\
3-4 \\
(n=40)\end{array}$ & $\begin{array}{l}\text { Weiterbildungsjahr } \\
5-6 \\
(\mathrm{n}=33)\end{array}$ \\
\hline Sensitivität & $\begin{array}{l}100 \% \\
(\mathrm{KI} 15,81-100 \%)\end{array}$ & $\begin{array}{l}100 \% \\
(\mathrm{KI} 47,82-100 \%)\end{array}$ & $\begin{array}{l}100 \% \\
(\mathrm{KI} 29,24-100 \%)\end{array}$ \\
\hline Spezifität & $\begin{array}{l}96 \% \\
(\mathrm{KI} 79,65-99,99 \%)\end{array}$ & $\begin{array}{l}91,43 \% \\
(\mathrm{KI} 76,94-98,2 \%)\end{array}$ & $\begin{array}{l}97,06 \% \\
(\mathrm{KI} 84,67-99,93 \%)\end{array}$ \\
\hline $\begin{array}{l}\text { positiver prädiktiver } \\
\text { Wert }\end{array}$ & $\begin{array}{l}66,67 \% \\
(\mathrm{KI} 9,43-99,16 \%)\end{array}$ & $\begin{array}{l}62,5 \% \\
(\mathrm{KI} 24,49-91,48 \%)\end{array}$ & $\begin{array}{l}75 \% \\
(\text { KI 19,41-99,37\%) }\end{array}$ \\
\hline $\begin{array}{l}\text { negativer prädikti- } \\
\text { ver Wert }\end{array}$ & $\begin{array}{l}100 \% \\
(\mathrm{KI} 85,75-100 \%)\end{array}$ & $\begin{array}{l}100 \% \\
(\mathrm{KI} 89,11-100 \%)\end{array}$ & $\begin{array}{l}100 \% \\
(\mathrm{KI} 89,42-100 \%)\end{array}$ \\
\hline LR+ & $\begin{array}{l}25 \\
(\mathrm{KI} 3,66-170,59 \%)\end{array}$ & $\begin{array}{l}11,67 \\
\text { (KI 3,95-34,42 \%) }\end{array}$ & $\begin{array}{l}34 \\
\text { (KI 4,93-234,47 \%) }\end{array}$ \\
\hline LR- & 0 & 0 & 0 \\
\hline
\end{tabular}

Verwendete Abkürzungen: KI: Konfidenzintervall, LR: Likelihood Ratio.

Im zweiten Schritt wurde der Einfluss des Ausbildungsgrades auf die Dauer der Untersuchung analysiert und grafisch im folgenden Boxplot dargestellt (Abb. 15). Es wurden keine Diskrepanzen zwischen den drei Gruppen beobachtet. Mit Hilfe des Wilcoxon-Vorzeichen-Rang-Tests zeigte sich bei einem errechneten p-Wert von 0,95 kein signifikanter Unterschied zwischen den Gruppen.

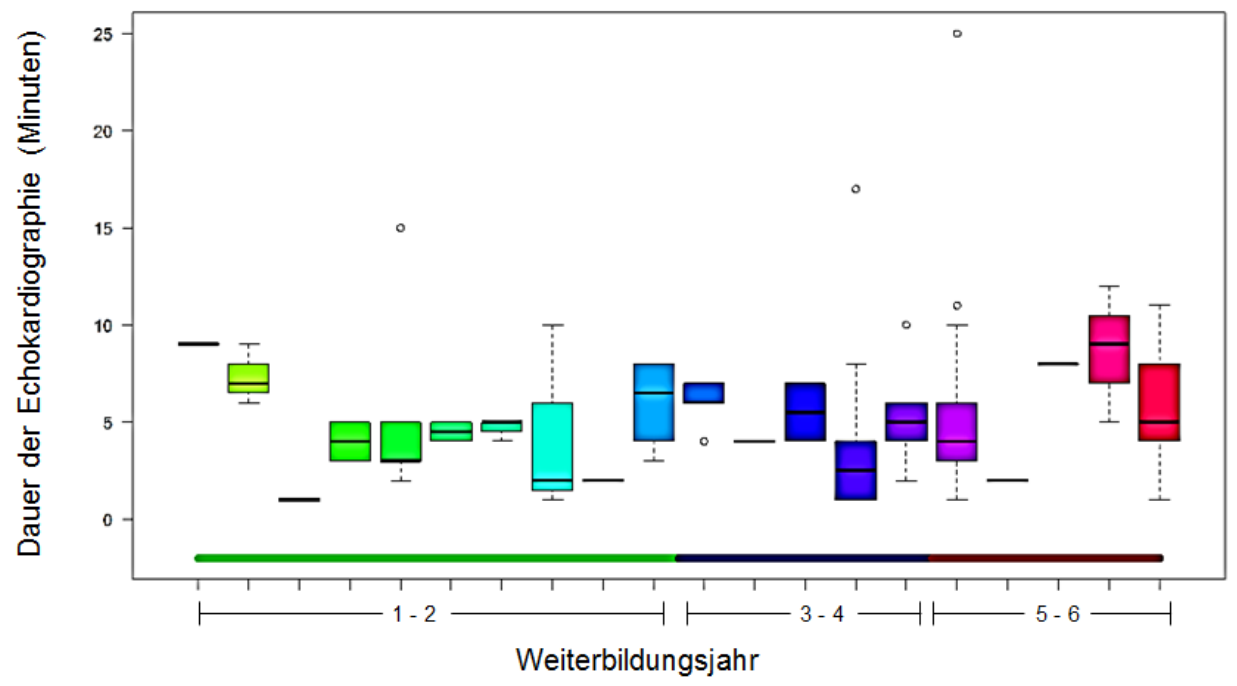

Abb. 15: Boxplot zur Darstellung des Einflusses des Weiterbildungsjahres auf die Dauer der Echokardiographie. 


\subsection{BMI und Dauer der Echokardiographie}

Weiterhin wurde der Body-Mass-Index als potentielle Einflussgröße auf die Dauer der Echokardiographie untersucht. Der BMI wurde aus der folgenden Formel berechnet:

$$
\mathrm{BMI}=\frac{\text { Körpergewicht }[\mathrm{kg}]}{(\text { Körpergröße }[\mathrm{m}])^{2}}
$$

Der mittlere BMI des gesamten Patientenkollektivs betrug $25,51 \mathrm{~kg} /(\mathrm{m})^{2}(\mathrm{SD}=$ 5,484404). Die mittlere Dauer für die Durchführung und Auswertung der fokussierten Echokardiographie lag, wie in Abschnitt 4.6 erwähnt, bei fünf Minuten. Nach Analyse der Korrelation zwischen den beiden Messgrößen resultierte ein nicht signifikanter Zusammenhang mit einem Korrelationskoeffizient nach Pearson von 0,1094 (95 \% KI $=[-0,0889,0,2994])$, die Dauer der Ultraschalluntersuchung wird nicht durch den BMI des Patienten beeinflusst (Abb. 16).

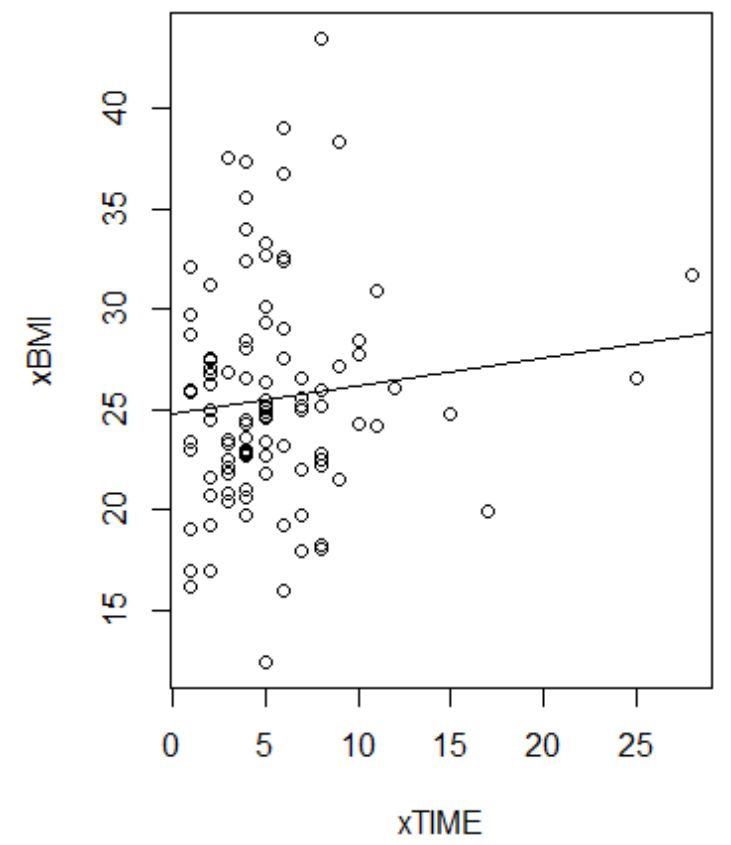

Abb. 16: Streudiagramm zur Darstellung des Einflusses des BMI auf die Dauer der Echokardiographie. Verwendete Abkürzungen: BMI: Body-Mass-Index.

\subsection{Komplikationen}

Im gesamten Patientengut trat ein Pneumothorax (1\%) auf, der sich im weiteren Verlauf spontan größenregredient zeigte. Der Patient blieb beschwerdefrei. Bei zwölf Katheteranlagen (12\%) kam es zu Fehllagen, die während der Durchführung der Echokardiographie detektiert wurden. In meisten Fällen handelte es sich um eine zu tiefe Positionierung im rechten Vorhof. Die jeweiligen Katheter wurden dann zurückgezogen bis ein sofortiger flush dargestellt werden konnte. Bei einem Patienten mit verspätetem flush-Test zeigte sich die Katheterspitze in Projektion auf die Aorta thoracalis descendens. Im CT des Thorax stellte sich eine persistierende linke V. cava superior dar, so dass der Katheter sofort entfernt wurde (Abb. 17). In einem Fall zeigte sich der flush verspätet mit einem retrograden Fluss vom rechten Ventrikel 
zum rechten Vorhof. Nach dem Rückzug des Katheters um 2,5 cm konnte ein sofortiger und orthograder flush detektiert werden (Abb. 18). Komplikationen durch die fokussierte Echokardiographie traten nicht auf.

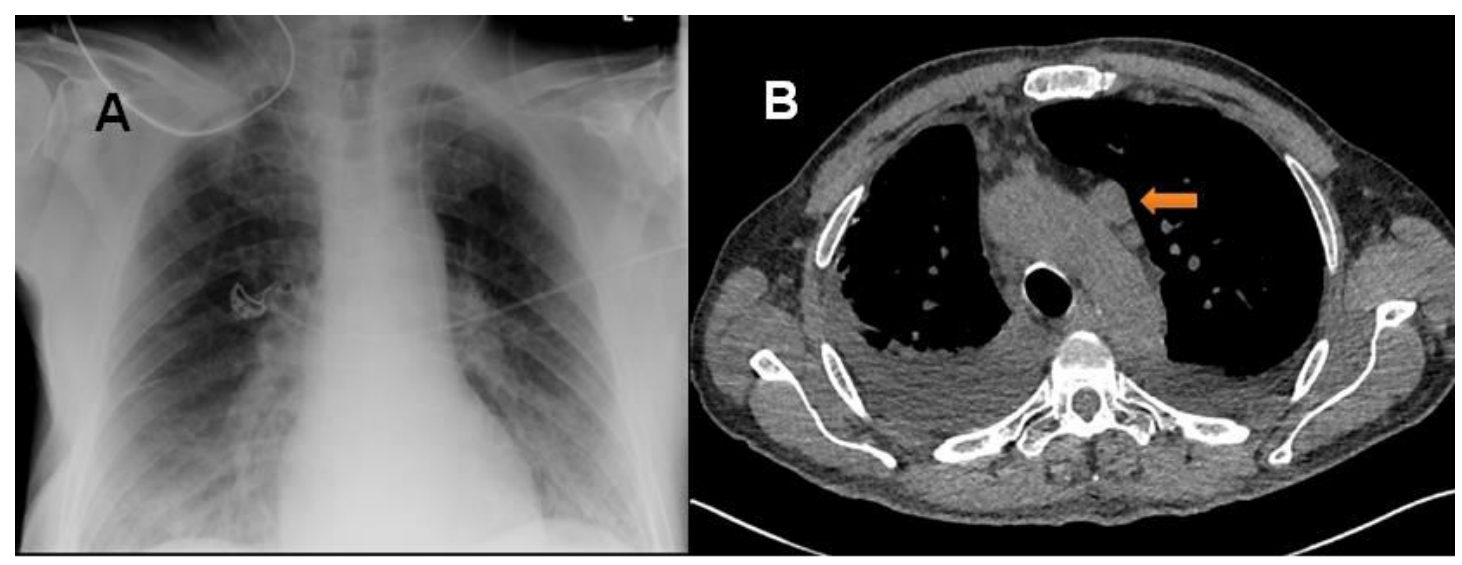

Abb. 17: Persistierende linke Vena cava superior im Röntgen-Thorax (A) und im CT (B, Pfeil).

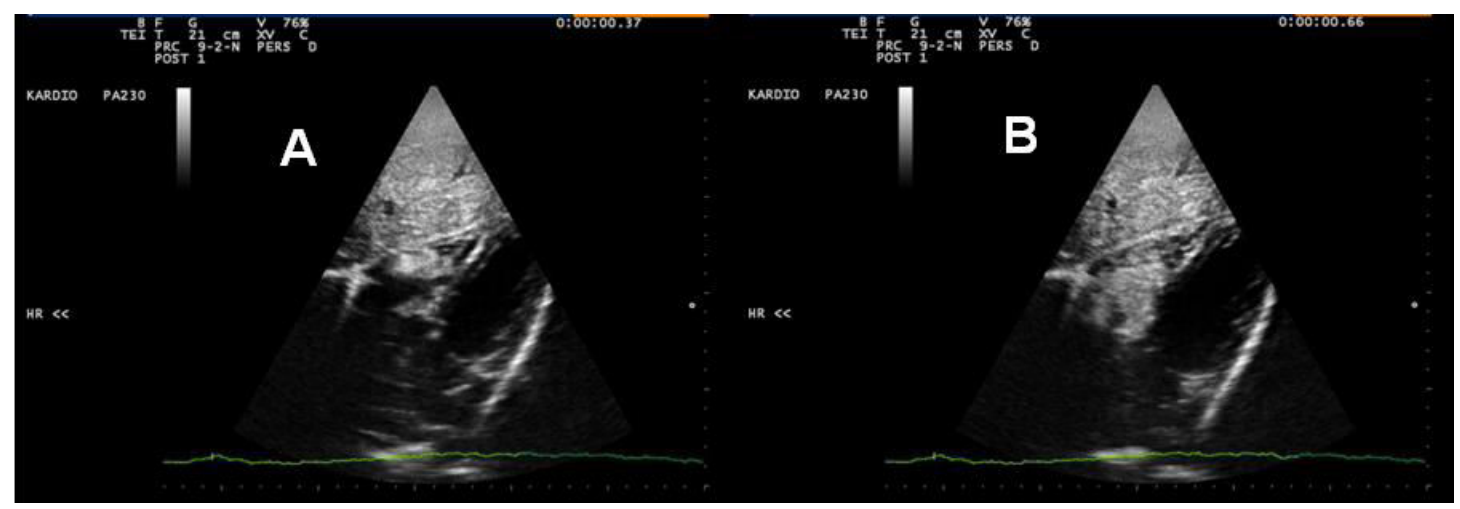

Abb. 18: Fehllage des zentralvenösen Katheters im rechten Ventrikel mit retrogradem flush. (A: Beginn des Tests mit Kontrastierung des rechten Ventrikels, B: Retrograder Fluss vom Ventrikel in den rechten Vorhof). 
Die Anlage eines zentralvenösen Katheters gehört zur Standardprozedur in der Notfall- und Intensivmedizin. Sie dient der Gabe von Flüssigkeit, Medikamenten und Ernährung sowie der hämodynamischen Überwachung. Weitere Anwendungsbereiche sind die Hämofiltration / Hämodialyse und interventionelle Maßnahmen, wie zum Beispiel ein temporärer Schrittmacher und das intravaskuläre Temperaturmanagement.

Die favorisierten Zugangswege sind die $\mathrm{Vv}$. jugulares internae, subclaviae und femorales, die entweder ultraschallgesteuert oder nach anatomischen Landmarken punktiert werden können. Durch die Anwendung des Ultraschalls wurde eine signifikante Reduktion von Komplikationen, wie arterielle Fehlpunktionen, Nachblutungen oder die Entstehung eines Pneumothorax erreicht (Jenssen et al. 2016).

Die ideale Lage der Katheterspitze ist oberhalb der Mündung der Vena cava superior in den rechten Vorhof und außerhalb des Perikardiums (Hsu et al. 2006; Smith und Nolan 2013). Durch eine Fehllage können schwerwiegende Komplikationen auftreten, wie z. B. Herzperforation oder Perikardtamponade (Collier und Goodman 1995; Booth et al. 2001), so dass vor der Verwendung des Katheters eine Lagekontrolle unbedingt erfolgen soll.

Auf der ICU- und IMC-Station unserer Klinik wurden im Jahr 2015 jeweils 954 und 1.222 Patienten behandelt. Bei ca. 90\% der Patienten auf der Intensivstation und bei ca. 50\% auf der IMC-Station wurden zentralvenöse Katheter gelegt und somit ungefähr 1.469 Katheter pro Jahr. Für die Detektion der Spitze des Katheters gibt es bisher keine Richtlinie und kein etabliertes Verfahren, welches eine korrekte Position verifizieren kann. Auf den meisten Intermediate Care- und Intensivstationen gilt eine Röntgen-Thoraxaufnahme als der Gold-Standard für die Lagekontrolle und den Ausschluss von postprozeduralen Komplikationen. Es gibt jedoch Zweifel daran, dass die Carina tracheae tatsächlich der Höhe der V. cava superior entspricht. Wirsing und Mitarbeiter zeigten mit Hilfe der transösophagealen Echokardiographie, dass eine Distanz bis $55 \mathrm{~mm}$ unterhalb der Carina toleriert werden kann und eine extraatriale Lage sichern kann (Wirsing et al. 2008). Daraufhin wurden weitere Techniken, wie die intravasale EKG-Ableitung (Lee et al. 2009), die Fluoroskopie (Ahn et al. 2012) oder die transthorakale Echokardiographie mit Darstellung des Führungsdrahtes in der V. cava inferior oder im rechten Atrium (Bedel et al. 2013) untersucht. Solche Verfahren sind jedoch eher unzuverlässig, zeitaufwendig oder auch potentiell gefährlich für die Patienten.

Im Jahr 2010 wurde eine neue sonographische Methode mit Hilfe von Kontrastmittel veröffentlicht. In der prospektiven Studie von Vezzani et al. wurden nach Anlage von insgesamt 111 Kathetern die Vv. subclaviae und Vv. jugulares sowie die Herzhöhlen mittels B-Mode untersucht. Anschließend wurden $9 \mathrm{ml}$ physiologischer Kochsalzlösung gemischt mit $1 \mathrm{ml}$ Luft über den Katheter appliziert. Das Vorhandensein einer Verwirbelung am Übergang der V. cava superior in den rechten Vorhof innerhalb von 1 bis 2 Sekunden wurde als Indikator einer korrekten Position gewertet. Anschließend erfolgte eine Thoraxsonographie zwecks Ausschluss eines Pneumothorax. Als Referenzmethode wurde das Röntgen-Thorax herangezogen. Die Methode zeigte 
jeweils eine hohe Sensitivität (96\%) und Spezifität (93\%) sowie einen signifikanten zeitlichen Vorteil des Ultraschalls mit 10 Minuten gegenüber dem Röntgen mit 83 Minuten. Ein Analyse der Kosten zeigte, dass die Sonographie um 2,81€ preisgünstiger als das Röntgen ist (Vezzani et al. 2010). Im Fallbericht von Prekker et al. wurde nur 0,9\%-ige Kochsalzlösung verabreicht mit einer vergleichbar präzisen Darstellung der Verwirbelung in der Echokardiographie (Prekker et al. 2010). Die neue Methode wurde auch bei Kindern mit zentralvenösen Kathetern in den Femoralgefäßen in einem Herzkatheterlabor untersucht. Ein positiver flush-Test sicherte, dass der Katheter im venösen System lag (Horowitz et al. 2014).

Im Jahr 2014 wurde eine prospektive Studie mit 152 ZVK bei 147 Patienten von Weekes et al. publiziert. Bei drei von vier fehlpositionierten Kathetern war der flushTest abwesend. Der vierte Katheter wurde über die rechte $\mathrm{V}$. subclavia eingebracht, die Spitze lag in der V. brachiocephalica. Der flush-Test zeigte sich positiv, was die Sensitivität auf $75 \%$ senkte. Die Spezifität betrug 100\%, der PPV 100\% und NPV 99,24\% (Weekes et al. 2014).

In weiteren Studien wurde gezeigt, dass die neue Methode eine hohe Präzision für die ZVK-Lagekontrolle aufweist (Weekes et al. 2014; Wen et al. 2014; Duran-Gehring et al. 2015; Gekle et al. 2015; Meggiolaro et al. 2015; Weekes et al. 2015).

In der Tabelle 12 werden alle publizierten Arbeiten mit ihren Merkmalen zusammengefasst. 
Tab. 12: Übersicht der publizierten Studien und ihrer Merkmale.

Verwendete Abkürzungen: N/A: not available, US: Ultraschall

\begin{tabular}{|c|c|c|c|c|c|}
\hline Studie & Jahr & Journal & Studiendesign & Ausbildungsgrad der Ärzte & Patientenzahl \\
\hline Vezzani & 2010 & Critical Care Medicine & Prospektive Observationsstudie & Einzelner Intensivmediziner & 111 \\
\hline $\begin{array}{l}\text { Campo Dell } \\
\text { Orto }\end{array}$ & 2013 & European Heart Journal & Prospektive Observationsstudie & N/A & 95 \\
\hline Weekes & 2014 & Academic Emergency Medicine & Prospektive Observationsstudie & Fünf Intensivmediziner & 142 \\
\hline Wen & 2014 & Critical Care Medicine & Retrospektive Vergleichsstudie & Ärzte vom 1. bis 10 . Weiterbildungsjahr & 219 \\
\hline Baviskar & 2015 & $\begin{array}{l}\text { Indian Journal of Critical Care Medi- } \\
\text { cine }\end{array}$ & Prospektive Observationsstudie & US-erfahrener Intensivmediziner & 25 \\
\hline Duran-Gehring & 2015 & $\begin{array}{l}\text { The American Journal of Emergency } \\
\text { Medicine }\end{array}$ & Prospektive Observationsstudie & $\begin{array}{l}\text { Zwei Intensivmediziner mit } 1 \text { Monat Rota- } \\
\text { tion im US }\end{array}$ & 46 \\
\hline Gekle & 2015 & Journal of Ultrasound in Medicine & Prospektive Observationsstudie & $\mathrm{N} / \mathrm{A}$ & 68 \\
\hline Meggiolaro & 2015 & Minerva Anestesiologica & Prospektive Observationsstudie & $\begin{array}{l}\text { Ärzte mit 3-jähriger Erfahrung im vaskulä- } \\
\text { ren US und Echokardiographie }\end{array}$ & 105 \\
\hline Weekes & 2016 & Emergency Medicine Journal & Prospektive Observationsstudie & Studienärzte & 151 \\
\hline
\end{tabular}


In der hier vorliegenden Arbeit wurde die Lagekontrolle des ZVK mit Hilfe der fokussierten Echokardiographie nach Kochsalzinjektion über den Katheter analysiert. Wir führten eine prospektive, monozentrische klinische Studie mit insgesamt 100 Patienten durch. Der primäre Endpunkt war die Evaluation der Effektivität der fokussierten Echokardiographie im Vergleich zum Röntgen. Um die neue Methode zu testen, wurden die Patienten in zwei Gruppen eingeteilt: eine Testgruppe mit den ersten 10 Patienten, die vom erfahrenen Studienarzt untersucht wurden und eine Validierungsgruppe mit den restlichen 90 Patienten, die von den diensthabenden Ärzten der Stationen eingeschlossen wurden. Die postprozeduralen Röntgen-Thoraxaufnahmen wurden in a.p.-Projektion im Liegen durchgeführt und vom unabhängigen Untersucher und einem verblindeten Radiologen befundet. Nach dem Aufsuchen der Carina tracheae erfolgte die Unterteilung in vier radiologische Zonen (Abb. 12). In beiden Gruppen wurde in $74 \%$ der Fälle eine korrekte Position dokumentiert, $3 \%$ lagen zu hoch in der Zone C und 23 \% zu tief im rechten Vorhof. Die Notwendigkeit zur Lagekorrektur wurde vom Punkteur ermessen und durchgeführt.

In der Bewertung der Röntgenaufnahmen von zwei unabhängigen Untersuchern wurde kein signifikanter Unterschied beobachtet. Für die fokussierte Echokardiographie wurde in den meisten Fällen (86 \%) der subkostale Vierkammerblick angewendet. Im Gesamtkollektiv lagen $83 \%$ der Katheter beim positiven flush-Test (RASS rapid atrial swirl sign, weniger als zwei Sekunden nach Injektion) korrekt. Somit konnte eine Spezifität von $94,32 \%$ in beiden Kohorten errechnet werden. Diese bringt zum Ausdruck, dass bei Nachweis des RASS die Katheterspitze mit 94 \%-iger Wahrscheinlichkeit in der richtigen Position liegt. Im klinischen Umfeld lässt sich schlussfolgern, dass beim positiven RASS keine Röntgenaufnahme benötigt wird. Die Sensitivität betrug 100\% (Wahrscheinlichkeit, dass bei negativem RASS der Katheter nicht korrekt liegt). In 17\% wurde anhand der Echokardiographie eine Fehllage dokumentiert, wobei in fünf Fällen der Katheter laut Röntgen korrekt lag. Das kann einen Zusammenhang mit schwierigen anatomischen Verhältnissen des Patienten haben und setzt daher eine gute Bildqualität voraus. Die Übereinstimmung für die Befundung der fokussierten Echokardiographie zwischen zwei unabhängigen Untersuchern wurde in unserer Studie analysiert und zeigte eine gute interrater-Reliabilität, die die Zuverlässigkeit der Bewertungen zum Ausdruck bringt.

In der Studie von Vezzani et al. (2010) zeigte die neue Methode ebenfalls eine hohe Sensitivität (96\%) und Spezifität (93\%) und die Sonographie war mit 2,81 € preisgünstiger als das Röntgen (Vezzani et al. 2010). In einer ähnlichen Arbeit von Weekes et al. (2014) mit 152 ZVKs bei 142 Patienten wurde eine geringere Sensitivität von $75 \%$ berechnet. Grund dafür war die kleine Anzahl der vier malpositionierten Katheter mit abwesendem flush-Test in dreien davon. Trotzdem blieb die Spezifität mit 100\% eindrucksvoll hoch; der PPV betrug 100\%, der NPV 99,24 \% (Weekes et al. 2014). Laut Baviskar et al. (2015) kann die neue Methode eine Sensitivität und Spezifität von $100 \%$ erreichen, die durchgeführte Studie weist jedoch eine schlechte Qualität aus, da die Untersuchungen in nur 25 Patienten mit einem Linear-Schallkopf 10-5 MHz gemacht wurden (Baviskar et al. 2015). In der prospektiven Arbeit von Duran-Gehring et al. (2015) in 46 Patienten wurden drei Fehllagen mit verspätetem oder abwesendem flush detektiert (Duran-Gehring et al. 2015). Meggiolaro et al. (2015) präsentierten ebenfalls akkurate Ergebnisse mit jedoch verschärftem zeitli- 
chem Abstand zwischen der Injektion der Kochsalzlösung und der sichtbaren Verwirbelung auf $500 \mathrm{~ms}$, was zu einer Sensitivität von $10 \%$ und Spezifität von 99\% geführt hat (Meggiolaro et al. 2015).

Unsere Ergebnisse zeigen, dass die fokussierte Echokardiographie mit fünf Minuten einen signifikant geringeren Zeitaufwand als die Röntgenaufnahmen aufweist, die durchschnittlich 49,5 Minuten dauerten. Das korreliert gut mit Resultaten aus anderen Studien, die einen wesentlichen zeitlichen Vorteil des Ultraschalls nachweisen. Wen et al. haben 202 Patienten mit insgesamt 219 Dialysekathetern retrospektiv analysiert und beschrieben einen durchschnittlichen Zeitabstand für das Röntgen von 28,3 Minuten (10-360 Minuten) und für den Ultraschall von 3,2 Minuten (2-5 Minuten) (Wen et al. 2014). Vezzani et al. (2010), Duran-Gehring et al. (2014), Gekle et al. (2015) und Weekes et al. (2016) führten zusätzlich eine Thoraxsonographie zum Ausschluss eines Pneumothorax durch. Beide Untersuchungen dauerten $10 \pm 5 \mathrm{Mi}-$ nuten (3-20 Minuten) im Vergleich zum Röntgen mit $83 \pm 79$ Minuten (15-368 Minuten) (Vezzani et al. 2010). Duran-Gehring et al. (2014) veröffentlichten kürzeren Zeiten von 5 Minuten (4,2-5,9 Minuten) für den Ultraschall und 28.2 Minuten (16,8-39,4 Minuten) für das Röntgen (Duran-Gehring et al. 2015). In der Arbeit von Gekle et al. (2015) wurden ähnliche Ergebnisse publiziert mit einer Dauer für die Sonographie von 8,8 Minuten (7,46-10,14 Minuten) und für die Thoraxaufnahme von 45,78 Minuten (37,03-54,54 Minuten) (Gekle et al. 2015). Der zeitliche Unterschied zwischen beiden Untersuchungen in der Studie von Weekes et al. (2016) war 23,8 Minuten (19,6-29.3 Minuten) (Weekes et al. 2015). Mit Hilfe des Ultraschalls kann daher eine schnelle Verwendung des zentralvenösen Katheters erfolgen, die für die kritisch kranken Patienten von großer Bedeutung ist.

Tabelle 13 fasst die Ergebnisse der publizierten Studien zusammen.

Tab. 13: Ergebnisse der bereits publizierten Studien.

Verwendete Abkürzungen: N/A: not available, Min.: Minute, Sek.: Sekunde.

\begin{tabular}{|l|l|l|l|l|l|}
\hline Studie & Jahr & Sensitivität & Spezifität & Dauer des US & Dauer des Röntgens \\
\hline Vezzani & 2010 & $96 \%$ & $93 \%$ & $10 \pm 5(3-20)$ Min. & $83 \pm 79(15-368)$ Min. \\
\hline $\begin{array}{l}\text { Campo Dell } \\
\text { Orto }\end{array}$ & 2013 & N/A & $100 \%$ & N/A & N/A \\
\hline Weekes & 2014 & $75 \%$ & $100 \%$ & N/A & N/A \\
\hline Wen & 2014 & N/A & N/A & $3,2(2-5)$ Min. & $28,3(10-360)$ Min. \\
\hline Baviskar & 2015 & $100 \%$ & $100 \%$ & $30-60$ Sek. & 20 bis >60 Min. \\
\hline $\begin{array}{l}\text { Duran- } \\
\text { Gehring }\end{array}$ & 2015 & N/A & N/A & $5(4,2-5,9)$ Min. & $28.2(16,8-39,4)$ Min. \\
\hline Gekle & 2015 & N/A & N/A & $8,8(7,46-10,14)$ Min. & $45,78(37,03-54,54)$ Min. \\
\hline Meggiolaro & 2015 & $64 \%$ & $100 \%$ & $5(5-10)$ Min. & $67(42-84)$ Min. \\
\hline Weekes & 2016 & N/A & N/A & 1,1 Min. & 20 Min. \\
\hline
\end{tabular}

Ein zusätzlicher Vorteil der Sonographie ist die fehlende Strahlenbelastung des Patienten. Für die aus unnatürlichen Quellen stammende Strahlenexposition ist haupt- 
sächlich die diagnostische Medizin verantwortlich. Das Ausmaß des Krebsrisikos durch medizinische Strahlenbelastung wurde im Jahr 2004 in 14 Ländern untersucht. Den zweiten Platz belegte Deutschland mit einem geschätzten das Risiko von 1,5\% und somit 2.049 zusätzlichen Krebserkrankungen pro Jahr (de Gonzalez und Darby 2004). Die Minimierung der Strahlenexposition muss daher immer gefordert werden und wird von der Strahlenschutzverordnung (StrlSchV) unterstützt: $\S 81$ Abs. 1 ,Die durch ärztliche Untersuchungen bedingte Strahlenexposition ist so weit einzuschränken, wie dies mit den Erfordernissen der medizinischen Wissenschaft zu vereinbaren ist." (Strahlenschutzverordnung vom 20. Juli 2001)

Idealerweise sollte die Prozedur einfach zu erlernen sein, daher wurde als sekundärer Endpunkt das Leistungsniveau der diensthabenden Ärzte aus verschiedenen Weiterbildungsjahren geprüft, was in den bisher publizierten Studien nicht untersucht wurde. Um den sekundären Endpunkt zu analysieren, wurde die fokussierte Echokardiographie durch insgesamt 22 Assistenzärzte aus verschiedenen Weiterbildungsjahren durchgeführt. Die Testcharakteristika sowie die Dauer der Untersuchung zeigten keinen signifikanten Unterschied zwischen den Gruppen. In Anbetracht diesen Ergebnissen erscheint dieses Verfahren nach kurzem Training einfach erlernbar zu sein. Schließlich wurde die Korrelation zwischen dem Body-Mass-Index und der Dauer der Echokardiographie untersucht, mit Ausschluss eines relevanten Einflusses.

Unsere Studie weist einige Limitationen auf. Zunächst wäre die geringe Erfahrung der Assistenzärzte in der Notfallsonographie zu erwähnen, die Kolleginnen / -en wurden nur einem 30- bis 60-minütigen Training durch die Studienärzte unterzogen. In unserer Klinik und in vielen Institutionen in Deutschland gibt es jedoch keine Regelausbildung für die fokussierte Notfallsonographie, sodass unsere Ergebnisse einen pragmatischen Ansatz widerspiegeln. In der Beobachtungsstudie von Breitkreutz et al. konnte die fokussierte Echokardiographie im Rahmen der kardiopulmonalen Reanimation nach einem 8-stündigen Training qualitativ hochwertig von Anfängern durchgeführt werden (Breitkreutz et al. 2009).

Der zweite mögliche Kritikpunkt an unserer Studie ist die nicht erfolgte Thoraxsonographie zwecks Ausschluss eines Pneumothorax. Vezzani et al. hingegen haben diesen Aspekt untersucht. Die Detektion des Pneumothorax erfordert ein umfangreicheres Training und war nicht das Ziel unserer Arbeit.

Der dritte Kritikpunkt betrifft die sonographische Kontrolle der Vena jugularis und Vena subclavia zum Ausschluss einer Kathetermigration, wie in der Arbeit von Maury et al. beschrieben. Dieses Vorgehen könnte theoretisch nach einem negativen flushTest sinnvoll sein. Unser primäres Ziel war jedoch der Vergleich der fokussierten Echokardiographie mit dem Röntgen und die Beurteilung des Leistungsniveaus der Weiterbildungsassistenten nach dem kurzen Training, so dass diese Untersuchung explizit nicht durchgeführt wurde.

Im gesamten Patientenkollektiv erfolgte die Durchführung der fokussierten Echokardiographie komplikationslos. In einem Fall wurde radiologisch ein Mantelpneumothorax festgestellt, der sich von selbst zurückbildete. Eine Fehllage wurde in 12 Fällen $(12 \%)$ mit Hilfe des Röntgens und des flush-Tests erkannt. Im Großteil lagen die 
Spitzen zu tief im rechten Vorhof und wurden dann vom Punkteur zurückgezogen. Bei einem Katheter konnte echokardiographisch sehr eindrücklich eine intraventrikuläre Lage dargestellt werden, als der flush retrograd vom Ventrikel in den Vorhof ausgerichtet war (Abb. 18). In einem anderen Fall stellte sich die Katheterspitze in Projektion auf die Aorta thoracalis descendens dar und der flush-Test war verspätet. Im nachfolgenden CT des Thorax wurde eine persistierende linke Vena cava superior diagnostiziert. Wegen der erhöhten Gefahr für Herzrhythmusstörungen (Morgan et al. 2015) wurde der Katheter sofort entfernt. 


\section{Zusammenfassung}

Einleitung: Die Anlage eines zentralvenösen Katheters gehört zu den StandardProzeduren im Bereich der Intensiv- und Notfallmedizin. Die ultraschallgestützte Anlage wird wegen geringerer Komplikationen allgemein empfohlen. Für die Lagekontrolle der Katheterspitze gibt es jedoch keinen Konsens darüber, welche Methode am besten geeignet ist. Eine neuartige und in wenigen Studien mit verschiedenen Settings untersuchte Methode ist die Darstellung eines flush (auch: rapid atrial swirl sign) nach Injektion eines $10 \mathrm{ml}$ Flüssigkeitsbolus.

Methodik: Wir führten eine prospektive klinische Studie mit insgesamt 100 Patienten der Intermediate Care- (IMC-Station 1021) und Intensivstation (ICU-Station 1022) der Klinik für Nephrologie und Rheumatologie durch. Die ersten zehn Patienten (Testkohorte) wurden vom erfahrenen Studienarzt untersucht und die restlichen 90 Patienten (Validierungskohorte) von Assistenzärzten aus verschiedenen Weiterbildungsjahren. Die Anleitung und Ausbildung in der fokussierten Echokardiographie erfolgte nach einem 30- bis 60-minütigen Training durch die Studienärzte. Alle Patienten erhielten eine postprozedurale Röntgen-Thorax-Aufnahme als derzeitiger Goldstandard für die Lagekontrolle der Katheterspitze. Die Auswertung der Bilder erfolgte durch einen unabhängigen Untersucher und einen verblindeten Radiologen. Direkt nach der ZVK-

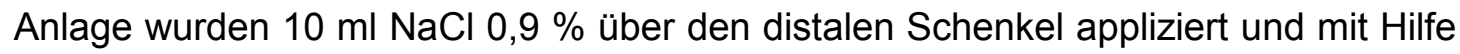
der fokussierten Echokardiographie die Darstellung des Flüssigkeitsbolus im Herzen beurteilt. Eine sofort sichtbare Verwirbelung (flush oder RASS) im rechten Vorhof zeigte sich innerhalb von zwei Sekunden nach Injektion und wurde als negativer screening-Test gewertet. Ein verspäteter (mehr als zwei Sekunden nach Injektion) oder abwesender flush wurden als positiver screening-Test für Fehllage definiert. Eine Stichprobe von $30 \%$ der Videoaufnahmen wurde von zwei unabhängigen Untersuchern evaluiert.

Ergebnisse: Die Sensitivität betrug 100\% (95\% KI 73,54-100\%). Die Spezifität betrug $94,32 \%$ (95\% KI 87,24-98,13\%) und variierte zwischen $91-97 \%$ in allen Gruppen der Weiterbildungsassistenten. Die positiven bzw. negativen prädiktiven Werte betrugen $70,59 \%(95 \% \mathrm{KI} 44,04-89,69 \%)$ und $100 \%$ (95\% KI 95,65-100\%) im gesamten Patientenkollektiv. Die mittlere Dauer für die Durchführung der fokussierten Echokardiographie betrug in beiden Kohorten 5 (1-28) Minuten und für das RöntgenThorax 49,5 (13-254) Minuten. Die Interrater-Reliabilität des RASS zeigte eine gute Übereinstimmung zwischen den Bewertern mit einem Cohen's-Kappa-Koeffizienten von 0,772 . Die Interpretation der Röntgenaufnahmen durch zwei unabhängige Untersucher ergab ebenfalls eine gute Korrelation $\left(r^{2}=0.8665\right)$. Die Testcharakteristika waren unabhängig vom Weiterbildungsjahr des Untersuchers.

Fazit: Die fokussierte Echokardiographie zur Darstellung des RASS für die Lagekontrolle der Katheterspitze hat eine ausgezeichnete Sensitivität und Spezifität und konnte gleichermaßen von erfahrenen und unerfahrenen Assistenzärzten durchgeführt werden. Die Ergebnisse legen nahe, dass die routinemäßige postprozedurale Röntgenaufnahme bei einem vorhandenen rapid atrial swirl sign nicht erforderlich ist. Dies würde eine Reduktion der Strahlenbelastung und der Kosten bewirken und zur Zeitersparnis führen. Ein negativer RASS sollte zu einer erweiterten sonographischen oder radiologischen Diagnostik führen. 
6. Anhang

6.1 Patienteninformation

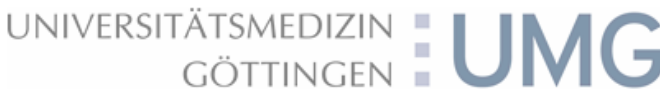 \\ B. Nephrologie \\ Rheumatologie}

Zentrum Innere Medizin Abteilung Nephrologie und Rheumatologie Ärztlicher Direktor Prof. Dr. med. Gerhard Anton Müller

\section{Information für Patienten}

\section{zur Studie}

\section{„Point of care Ultraschall versus Röntgen-Thorax zur Lagekontrolle zentralvenöser Katheter"}

Liebe Patientin, lieber Patient,

wir möchten Sie bitten, an einer wissenschaftlichen Studie teilzunehmen, die zur Verbesserung der medizinischen Versorgungsqualität beitragen soll.

Sie erhalten vorab eine ausführliche Information über das Wesen der Studie und nachfolgend die Gelegenheit, mit dem zuständigen Arzt offene Fragen zu besprechen. Wenn Sie den Inhalt der Studie und die möglichen Risiken verstanden haben, werden Sie gebeten, Ihr Einverständnis zur Studienteilnahme durch Ihre Unterschrift zu dokumentieren. Die Teilnahme an der Studie ist freiwillig und kann jederzeit ohne Angabe von Gründen widerrufen werden. Der spätere Widerruf der Teilnahme schließt die Vernichtung aller erhobenen Daten und die Löschung Ihrer Daten ein. Weder durch eine Nichtteilnahme noch durch einen späteren Widerruf der Teilnahme entstehen Ihnen irgendwelche Nachteile für die weitere Behandlung Ihrer Erkrankung.

Bei Ihnen wurde die Anlage eines zentralvenösen Katheters (ZVK) notwendig. Diese Katheter werden zur Infusion bestimmter Medikamente (z. B. kreislaufwirksame Medikamente) oder zur parenteralen Ernährung verwendet, da diese bei Gabe über herkömmliche Venenzugänge zu einer Venenentzündung führen

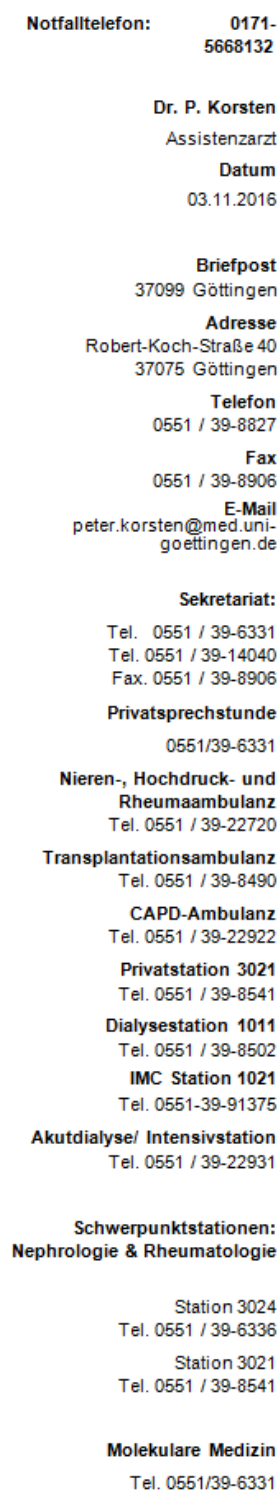


können. Auch die Notwendigkeit gehäufter Blutentnahmen, wie es beispielsweise bei intensivmedizinischer Behandlung erforderlich ist, stellt eine häufige Indikation zur Verwendung eines ZVK dar.

Bei der Anlage wird unter örtlicher Betäubung und mit Ultraschallunterstützung ein zentrales venöses Gefäß (üblicherweise eine der großen Venen im Hals- oder Schlüsselbeinbereich) aufgesucht und der Katheter über einen Führungsdraht in die Vene eingeführt. Dieses Verfahren ist ein langjährig etabliertes Routineverfahren.

Nach Beendigung des Eingriffes wird eine Röntgenaufnahme der Lunge (Thorax) durchgeführt, um die korrekte Lage des Katheters darzustellen.

Im Rahmen der Studie soll untersucht werden, ob mit Hilfe von Ultraschall und der Injektion von $10-20 \mathrm{~mL}$ Kochsalzlösung die Katheterlage auch ohne die Durchführung einer Röntgenaufnahme sicher kontrolliert werden kann. Hierzu wird das Herz von außen mit einer speziellen Ultraschallsonde aufgesucht und insbesonderes der rechte Herzvorhof, oberhalb dessen der Katheter liegt, aufgesucht. Nach Injektion von 10-20 mL Kochsalzlösung kommt es erwartungsgemäß zu einer Verwirbelung des Blutflusses, die sich im Ultraschallbild sehr gut nachweisen lässt.

Zu Vergleichszwecken wird - wie sonst auch üblich - eine Röntgenaufnahme der Lunge angefertigt und es werden Zeitaufwand und die Übereinstimmung beider Verfahren miteinander verglichen. Ziel der Studie ist es zu untersuchen, ob die Lagebestimmung des Katheters mittels Ultraschall möglich ist, was für die Patienten eine schnellere Anwendung von Medikamenten und ohne Strahlenbelastung bedeutet.

Die Untersuchung mittels Ultraschall ist nicht schmerzhaft und Sie werden keiner zusätzlichen Strahlenbelastung ausgesetzt. Auch die Verwendung von Kochsalzlösung ist im Rahmen des Eingriffs üblich, um den Katheter von Blutresten freizuspülen, die die Funktion des Katheters ansonsten gefährden könnten.

Um die Daten den jeweiligen Patienten zuordnen zu können, werden die folgenden Informationen in pseudonymisierter Form Ihrer Akte entnommen: Alter, Geschlecht, Ergebnis der Ultraschalluntersuchung sowie Röntgenaufnahme(n). Pseudonymisiert bedeutet, dass Ihre Daten verschlüsselt, d.h. keine Angaben von Namen oder Initialen verwendet werden, sondern nur ein Nummern- oder Buchstaben Code, evtl. mit Angabe des Geburtsjahres.

\section{Hinweise zum Datenschutz:}

Die genannten Daten werden in der Klinik für Nephrologie und Rheumatologie gesammelt und aufbewahrt bis zum Abschluss des Forschungsprojekts. Die erhobenen Daten werden gespeichert, eine Weiterleitung der Daten an Dritte ist nicht vorgesehen und darf nur in pseudonymisierter Form erfolgen. Daneben ist eine Rückverfolgung der Daten aus der Datenbank nur durch den betreuenden Arzt möglich und durch Dritte nicht erlaubt. Die Daten werden entsprechend den Regelungen des Datenschutzgesetzes aufbewahrt. Die Daten sollen abschließend in pseudonymisierter Form in wissenschaftlichen Veröffentlichungen verwendet werden. Die ärztliche Schweigepflicht gilt im Rahmen der

Studie uneingeschränkt.

Die personenbezogenen Daten werden nach Beendigung oder Abbruch der Prüfung mindestens 10 Jahre aufbewahrt. Danach werden diese Daten gelöscht, soweit nicht gesetzliche oder satzungsgemäße Aufbewahrungsfristen entgegenstehen.

Bei offenen Fragen wenden Sie sich bitte an:

Herrn Dr. med. Peter Korsten, Universitätsmedizin Göttingen, Klinik für Nephrologie und Rheumatologie Robert-Koch Str. 40, 37099 Göttingen, Tel. 0551-39-14146 oder

Frau Eirini Mavropoulou, Universitätsmedizin Göttingen, Klink für Gastroenterologie und gastrointestinale Onkologie Robert-Koch-Str. 40, 37099 Göttingen, Tel. 0551-39-8827 


\subsection{Einwilligungserklärung}

\section{UNIVERSITÄTSMEDIZIN $=\mathbf{W M G}$
GÖTTINGEN $=0$ \\ S. Nephrologie \\ Rheumatologie}

Universitätsmedizin Göttingen

Und Rheumatologie

Prof. Dr. G. A. Müller, Robert-Koch Str. 40,37075 Göttingen

Zentrum Innere Medizin Abteilung Nephrologie und Rheumatologie Ärztlicher Direktor Prof. Dr. med. Gerhard Anton Müller

Ich habe die Patienteninformation über die o.g. Studie gelesen und habe diese vollständig verstanden. Ich hatte dabei die Gelegenheit, noch offene Fragen mit dem aufklärenden Arzt zu meiner Zufriedenheit zu besprechen.

\section{Freiwilligkeit}

Die Teilnahme an der Studie ist freiwillig und kann jederzeit ohne Angabe von Gründen von mir widerrufen werden, ohne dass mir dadurch Nachteile erwachsen. Ich wurde darüber informiert, dass dies einschließt, dass jederzeit eine Löschung meiner Daten erfolgt.

Mir ist bekannt, dass im Rahmen dieses Forschungsvorhabens personenbezogenen Daten erhoben und in pseudonymisierter (verschlüsselter) Form aufgezeichnet und gespeichert werden. Die Datenspeicherzeit beträgt 10 Jahre. Die personenbezogenen
Sekretariat: Tel. $0551 / 39-6331$ Tel. $0551 / 39-14040$ Fax. 0551 / 39-8906 Privatsprechstunde 0551/39-6331

Nieren-, Hochdruck- und Rheumaambulanz
Tel. $0551 / 39-22720$ Transplantationsambulan Tel. 0551 / 39-8490 CAPD-Ambulanz Tel. 0551 / 39-22922 Privatstation 3021 Tel. $0551 / 39-8541$ Dialysestation 1011 Tel. 0551 / 39-8502 IMC Station 1021 Tel. 0551-39-91375 Akutdialyse/ Intensivstation Tel. 0551 / 39-22931

Apherese-Station 1024 Tel. 0551 / 39-8506

Schwerpunktstationen: Nephrologie \& Rheumatologie Tel. 0551/39-6336 Station 3023 Tel. $0551 / 39-22606$

Molekulare Medizin Tel. 0551/39-6331 
Daten werden nicht an Dritte weitergegeben. Ich weiß, dass ich mein Einverständnis zur Speicherung der personenbezogenen Daten jederzeit widerrufen kann. Im Falle des Widerrufs werden alle personenbezogenen Daten gelöscht.

\begin{tabular}{lll}
\hline Ort, Datum & Patient/in & Unterschrift \\
\hline Ort, Datum & Aufklärender Arzt & Unterschrift
\end{tabular}




\subsection{Nicht einwilligungsfähige Patienten}

\section{UNIVERSITÄTSMEDIZIN \\ GÖTTINGEN

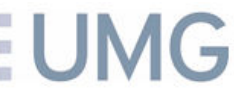 \\ 8. Nephrologie \\ Rheumatologie}

Tel. 0551 / 39-6331 Fax. 0551/39-8906

Privatsprechstunde 0551/39-6331 Nieren-, Hochdruck- und Rheumaambulanz
Tel. $0551 / 39-22720$ Transplantationsambulanz CAPD-Ambulanz Privatstation 3021 Privatstation 3021
Tel. $0551 / 39-8541$ Dialysestation 1011 Tel. 0551 / 39-8502 IMC Station 1021 Tel. 0551-39-91375 Akutdialyse/ Intensivstation Tel. 0551 / 39-22931 Apherese-Station 1024 Tel. 0551 / 39-8506 Schwerpunktstationen: Nephrologie \& Rheumatologie Tel. $0551 / 39-6336$ Station 3023 Tel. 0551 / 39-22606

Ort, Datum

Arzt Unterschrift

Molekulare Medizin 


\subsection{Wiedererlagung der Einwilligungsfähigkeit}

Formular für die nicht-einwilligungsfähigen Patienten nach Wiedererlangung ihrer Einwilligungsfähigkeit

„Point of care Ultraschall versus Röntgen Thorax zur Lagekontrolle zentralvenöser Katheter"

Nach der Wiedererlagung meiner Einwilligungsfähigkeit wünsche ich mir:

$\square$ die weitere Teilnahme in der Studie.

$\square$ keine weitere Teilnahme in der Studie sowie die Löschung meiner Daten.

Ort, Datum

Patient Unterschrift

Ort, Datum

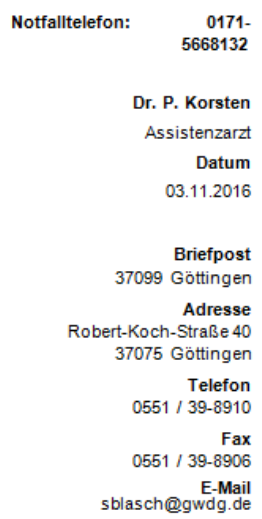

Tel. 0551 / 39-6331

Tel. $0551 / 39-14040$

Fax. 0551 / 39-8906

Privatsprechstunde

0551/39-6331

Nieren-, Hochdruck- und Rheumaambulan Tel. 0551 / 39-2272 Transplantationsambulan Tel. 0551 / 39-8490

CAPD-Ambulanz

Tel. 0551 / 39-22922

Privatstation 3021

Tel. 0551 / 39-854

Dialysestation 1011

Tel. 0551 / 39-8502

IMC Station 1021

Tel. 0551-39-91375 Akutdialyse/ Intensivstatio Tel. 0551 / 39-2293 Apherese-Station 1024

Schwerpunktstationen: Nephrologie \& Rheumatologie Station 302 Tel. 0551 / $39-6336$ Station 3023 Tel. 0551 / 39-2260

Molekulare Medizin Tel. 0551/39-6331 


\subsection{Untersuchungsprotokoll}

$\underline{\text { Patientenetikett }}$

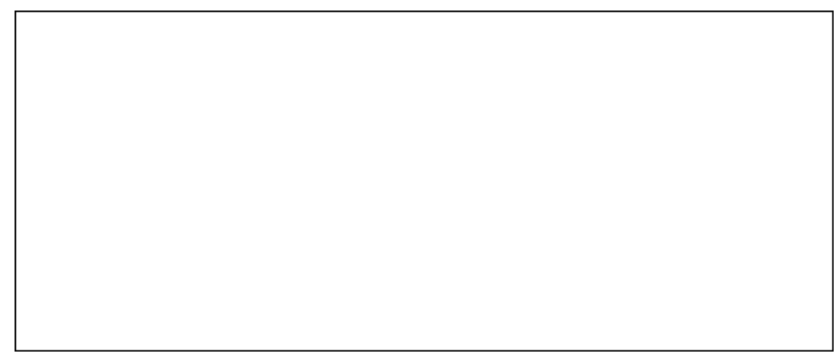

Datum der Untersuchung:

Station: $\quad \square$ IMC $1021 \quad \square$ ICU 1022

Größe: $\mathrm{cm}$

Gewicht: cm

RR: Systolisch: $\mathrm{mmHg}$ Diastolisch: $\mathrm{mmHg} \quad \mathrm{HF}$ : $/ \min$

Beatmung:

$\square$ keine

$\square$ nicht-invasiv

$\square$ invasiv

ZVK-Typ:

$\square$ 1-lumig $\square$ 2-lumig

$\square$ 3-lumig

$\square$ 4-lumig

$\square$ 5-lumig

Gefäß:

$\square$ V. jugularis interna

$\square$ V. subclavia

$\square$ rechts

$\square$ links

$\underline{\text { Tiefe: }}$ $\mathrm{cm}$

US-gesteuert:

$\square$ ja

$\square$ nein

Flush:

$\square$ sofortig (<2 sec)

$\square$ verspätet

$\square$ abwesend

Röntgen-Befund: $\quad \square$ innerhalb $3 \mathrm{~cm}$ oberhalb der Carina

$\square$ innerhalb $3 \mathrm{~cm}$ unterhalb der Carina

$\square$ Fehllage in:

Pneumothorax:

$\square$ ja

$\square$ nein

Zeitpunkt der ZVK-Anlage: hr Information an Radiologie: $\mathrm{hr}$

Zeitpunkt des Ultraschalls: $\mathrm{hr}$

Zeitpunkt des Röntgens: $\mathrm{hr}$

Untersucher: Weiterbildungsjahr: 


\subsection{Einwilligungserklärung für Abbildungen}

\section{UNIVERSITÄTSMEDIZIN GÖTTINGEN \#
GOG \\ S Nephrologie \\ Rheumatologie}

Zentrum Innere Medizin Abteilung Nephrologie und Rheumatologie Ärztlicher Direktor

\begin{tabular}{|l|l|}
\hline+2 \\
\hline
\end{tabular}
Prof. Dr. med. Gerhard Anton Müller

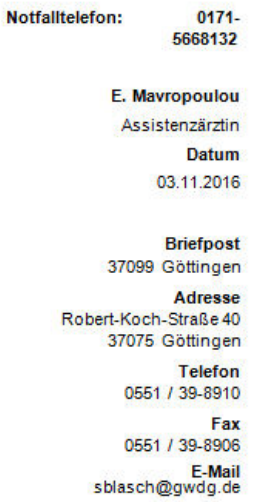

Einwilligungserklärung zur Veröffentlichung von personen-
bezogenen Abbildungen in der Dissertation mit Thema

„Point of care Ultraschall versus Röntgen Thorax zur Lagekontrolle zentralvenöser Katheter"

Ich wurde informiert dass meine persönlichen Fotografien ohne meinen Namen veröffentlicht werden und dass meine Anonymität Privatsprechstunde 0551/39-66331 garantiert werden kann. Meine Einwilligungserklärung kann ich jederzeit von der Veröffentlichung zurückziehen, allerdings nicht nach der Drucklegung der Dissertation.

Nieren-, Hochdruck- und Rheumaambulan
Tel. $0551 / 39-22720$ Transplantationsambulan Tel. 0551 / 39-8490 CAPD-Ambulan Tel. 0551 / 39-22922 Privatstation 3021 Tel. 0551 / 39-8541 Dialysestation 1011 Tel. 0551/39-8502 IMC Station 102 Tel. 0551-39-91375 Akutdialyse/ Intensivstation

Tel. 0551/39-8506

Schwerpunktstationen:

Ort, Datum Patient/in Unterschrift
Station 3024
Tel. $0551 / 39-66336$ Station 302 Tel. $0551 / 39-854$ Molekulare Medizin Tel. 0551/39-66331 


\section{Literaturverzeichnis}

Ahn SJ, Kim H-C, Chung JW, An SB, Yin YH, Jae HJ, Park JH (2012): Ultrasound and Fluoroscopy-Guided Placement of Central Venous Ports via Internal Jugular Vein: Retrospective Analysis of 1254 Port Implantations at a Single Center. Korean J Radiol 13, 314-323

Albrecht K, Nave H, Breitmeier D, Panning B, Tröger HD (2004): Applied anatomy of the superior vena cava-the carina as a landmark to guide central venous catheter placement. Br J Anaesth $\underline{92}, 75-77$

Aslamy Z, Dewald CL, Heffner JE (1998): MRI of Central Venous Anatomy: Implications for Central Venous Catheter Insertion. Chest J 114, 820-826

Aubaniac R (1952): L'injection intraveineuse sous-claviculaire; avantages et technique. Presse Med $\underline{60}, 1456$

Bailey SH, Shapiro SB, Mone MC, Saffle JR, Morris SE, Barton RG (2000): Is immediate chest radiograph necessary after central venous catheter placement in a surgical intensive care unit? Am J Surg $\underline{180}, 517-522$

Baskin JL, Pui C-H, Reiss U, Wilimas JA, Metzger ML, Ribeiro RC, Howard SC (2009): Management of occlusion and thrombosis associated with long-term indwelling central venous catheters. Lancet $\underline{374}, 159-169$

Baviskar AS, Khatib KI, Bhoi S, Galwankar SC, Dongare HC (2015): Confirmation of endovenous placement of central catheter using the ultrasonographic "bubble test". Indian J Crit Care Med 19, 38-41

Bedel J, Vallée F, Mari A, Riu B, Planquette B, Geeraerts T, Génestal M, Minville V, Fourcade $O$ (2013): Guidewire localization by transthoracic echocardiography during central venous catheter insertion: a periprocedural method to evaluate catheter placement. Intensive Care Med 39, 1932-1937

Bernard C: Leçons sur la chaleur animale, sur les effets de la chaleur et sur la fièvre. (Cours de médecine du Collége de France); Baillière, Paris 1876

Bleichroeder F, Unger E, Loeb W (1912): Intraarterielle Therapie. Berl Klin Wochenschr $\underline{49}, 1503-1506$

Booth SA, Norton B, Mulvey DA (2001): Central venous catheterization and fatal cardiac tamponade. Br J Anaesth 87, 298-302

Breitkreutz R, Uddin S, Steiger H, Ilper H, Steche M, Walcher F, Via G, Price S (2009): Focused echocardiography entry level: new concept of a 1-day training course. Minerva Anestesiol 75, 285-292

Campo Dell Orto M, Schellknecht S, Seeger FH, Hamm C, Breitkreutz R (2013): Sonographic position monitoring of central venous catheters by microbubbleinjection in real time: development of a new procedure. Eur Heart J $\underline{34}, \mathrm{P} 2944$

Chaney MA, Minhaj MM, Patel K, Muzic D (2007): Transoesophageal echocardiography and central line insertion. Ann Card Anaesth $\underline{10}, 127-131$ 
Chopra V, Anand S, Hickner A, Buist M, Rogers MA, Saint S, Flanders SA (2013a): Risk of venous thromboembolism associated with peripherally inserted central catheters: a systematic review and meta-analysis. Lancet $\underline{382}, 311-325$

Chopra V, O'Horo JC, Rogers MAM, Maki DG, Safdar N (2013b): The Risk of Bloodstream Infection Associated with Peripherally Inserted Central Catheters Compared with Central Venous Catheters in Adults: A Systematic Review and Meta-Analysis. Infect Control Hosp Epidemiol 34, 908-918

Chu KS, Hsu JH, Wang SS, Tang CS, Cheng KI, Wang CK, Wu JR (2004): Accurate Central Venous Port-A Catheter Placement: Intravenous Electrocardiography and Surface Landmark Techniques Compared by Using Transesophageal Echocardiography. Anesth Analg 98, 910-914

Collier P, Goodman G (1995): Cardiac tamponade caused by central venous catheter perforation of the heart: a preventable complication. J Am Coll Surg 181, 459-463

David JS, Tazarourte K, Perfus JP, Savary D (2005): Is ECG-guidance a helpful method to correctly position a central venous catheter during prehospital emergency care? Acta Anaesthesiol Scand 49, 1010-1014

Duran-Gehring PE, Guirgis FW, McKee KC, Goggans S, Tran H, Kalynych CJ, Wears RL (2015): The bubble study: ultrasound confirmation of central venous catheter placement. Am J Emerg Med 33, 315-319

Fletcher S, Bodenham A (2000): Safe placement of central venous catheters: where should the tip of the catheter lie? Br J Anaesth $\underline{85}, 188-191$

Forssmann W (1929): Die Sondierung des rechten Herzens. J Mol Med $\underline{8}, 2085-$ 2087

Forssmann W (1931): Über Kontrastdarstellung der Höhlen des lebenden rechten Herzens und der Lungenschlagader. Münch Med Wochenschr $\underline{78}$, 489-92

Frick H, Leonhardt H, Starck D: Spezielle Anatomie 2: Eingeweide, Nervensystem, Systematik der Muskeln und Leitungsbahnen. (Flexibles Taschenbuch: Med), 4., überarb. Auflage; Thieme, Stuttgart [u.a.] 1992

Gebhard RE, Szmuk P, Pivalizza EG, Melnikov V, Vogt C, Warters RD (2007): The Accuracy of Electrocardiogram-Controlled Central Line Placement: Anesth Analg $\underline{104}, 65-70$

Gekle R, Dubensky L, Haddad S, Bramante R, Cirilli A, Catlin T, Patel G, D'Amore J, Slesinger TL, Raio C, et al. (2015): Saline Flush Test: Can Bedside Sonography Replace Conventional Radiography for Confirmation of Above-theDiaphragm Central Venous Catheter Placement? J Ultrasound Med 34, 1295-1299

Gladwin MT, Slonim A, Landucci DL, Gutierrez DC, Cunnion RE (1999): Cannulation of the internal jugular vein: Is postprocedural chest radiography always necessary? Crit Care Med 27, 1819-1823

de Gonzalez AB, Darby S (2004): Risk of cancer from diagnostic X-rays: estimates for the UK and 14 other countries. Lancet $\underline{363}, 345-351$ 
Gravenstein N, Blackshear RH (1991): In vitro evaluation of relative perforating potential of central venous catheters: Comparison of materials, selected models, number of lumens, and angles of incidence to simulated membrane. J Clin Monit 므, 1-6

Guilbert M-C, Elkouri S, Bracco D, Corriveau MM, Beaudoin N, Dubois MJ, Bruneau L, Blair J-F (2008): Arterial trauma during central venous catheter insertion: Case series, review and proposed algorithm. J Vasc Surg 4ㅇ, 918-925

Hales S: Experiment 3. Statical Essays. (Haemastaticks); Innings Manby, London 1733

Hellerstein HK, Pritchard WH, Lewis RL (1949): Recording of Intracavity Potentials Through a Single Lumen, Saline Filled Cardiac Catheter. Exp Biol Med $\underline{71}$, 58-61

Horowitz R, Gossett JG, Bailitz J, Wax D, Pierce MC (2014): The FLUSH StudyFlush the Line and Ultrasound the Heart: Ultrasonographic Confirmation of Central Femoral Venous Line Placement. Ann Emerg Med 63, 678-683

Hsu JH, Wang CK, Chu KS, Cheng KI, Chuang HY, Jaw TS, Wu JR (2006): Comparison of radiographic landmarks and the echocardiographic SVC/RA junction in the positioning of long-term central venous catheters. Acta Anaesthesiol Scand $\underline{50}, 731-735$

Ingrande J, Lemmens HJ (2014): Medical devices for the anesthetist: current perspectives. Med Devices Auckl NZ $\underline{7}, 45-53$

Jenssen C, Brkljacic B, Hocke M, Ignee A, Piscaglia F, Radzina M, Sidhu PS, Dietrich CF (2016): EFSUMB Guidelines on Interventional Ultrasound (INVUS), Part VI - Ultrasound-Guided Vascular Interventions. Ultraschall Med - Eur J Ultrasound $\underline{37}, 473-476$

Kander T, Frigyesi A, Kjeldsen-Kragh J, Karlsson H, Rolander F, Schött U (2013): Bleeding complications after central line insertions: relevance of preprocedure coagulation tests and institutional transfusion policy. Acta Anaesthesiol Scand $\underline{57}, 573-579$

Kusminsky RE (2007): Complications of Central Venous Catheterization. J Am Coll Surg 204, 681-696

Lang H (2012): Zentralvenöse Zugänge - So bringen Sie den Katheter zum Herzen. Lege Artis - Mag Zur Ärztl Weiterbildung 2, 182-187

Lee J-H, Bahk J-H, Ryu H-G, Jung C-W, Jeon Y (2009): Comparison of the bedside central venous catheter placement techniques: landmark vs electrocardiogram guidance. Br J Anaesth 102, 662-666

Legler D, Nugent M (1984): Doppler Localization of the Internal Jugular Vein Facilitates Central Venous Cannulation. J Am Soc Anesthesiol 60, 481-482

Liu YT, Bahl A (2011): Evaluation of proper above-the-diaphragm central venous catheter placement: the saline flush test. Am J Emerg Med 29, 842 
Losert H, Prokesch R, Grabenwöger M, Waltl B, Apsner R, Sunder-Plassmann G, Muhm M (2000): Inadvertent transpericardial insertion of a central venous line with cardiac tamponade failure of preventive practices. Intensive Care Med $\underline{26}, 1147-1150$

Maki DG, Kluger DM, Crnich CJ (2006): The risk of bloodstream infection in adults with different intravascular devices: a systematic review of 200 published prospective studies. Mayo Clin Proc 81, 1159-1171.

Marik PE, Flemmer M, Harrison W (2012): The risk of catheter-related bloodstream infection with femoral venous catheters as compared to subclavian and internal jugular venous catheters: A systematic review of the literature and metaanalysis ${ }^{*}$. Crit Care Med 느, 2479-2485

Matsushima K, Frankel HL (2010): Bedside Ultrasound can Safely Eliminate the Need for Chest Radiographs after Central Venous Catheter Placement: CVC Sono in the Surgical ICU (SICU). J Surg Res $\underline{163}, 155-161$

Maury E, Guglielminotti J, Alzieu M, Guidet B, Offenstadt G (2001): Ultrasonic Examination: an alternative to chest radiography after central venous catheter insertion? Am J Respir Crit Care Med 164, 403-405

McGee DC, Gould MK (2003): Preventing Complications of Central Venous Catheterization. N Engl J Med 348, 1123-1133

Meggiolaro M, Scatto A, Zorzi A, Roman-Pognuz E, Lauro A, Passarella C, Bonaccorso G (2015): Confirmation of correct central venous catheter position in the pre-operative setting by echocardiographic "bubble test". Minerva Anestesiol 81, 989-1000

Meyers L (1945): Intravenous Catheterization. Am J Nurs 4토, 930-931

Molgaard O, Nielsen MS, Handberg BB, Jensen JM, Kjaergaard J, Juul N (2004): Routine X-ray control of upper central venous lines: Is it necessary? Acta Anaesthesiol Scand $\underline{48}, 685-689$

Morano SG, Coppola L, Latagliata R, Berneschi P, Chistolini A, Micozzi A, Girmenia C, Breccia M, Brunetti G, Massaro F, et al. (2014): Early and Late Complications Related to Central Venous Catheters in Hematological Malignancies: a Retrospective Analysis of 1102 Patients. Mediterr J Hematol Infect Dis $\underline{6}$, e2014011

Morgan LG, Gardner J, Calkins J (2015): The Incidental Finding of a Persistent Left Superior Vena Cava: Implications for Primary Care - Case and Review. Case Rep Med 2015, e198754

Moro ML, Viganò EF, Lepri AC, The Central Venous Catheter-Related Infections Study Group (1994): Risk Factors for Central Venous Catheter-Related Infections in Surgical and Intensive Care Units. Infect Control Hosp Epidemiol 15, 253-264

Müller MC, Arbous MS, Man S, M A, Vink R, Karakus A, Straat M, Binnekade JM, de Jonge E, Vroom MB, Juffermans NP (2015): Transfusion of fresh-frozen plasma in critically ill patients with a coagulopathy before invasive procedures: a randomized clinical trial (CME). Transfusion (Paris) $\underline{55}$, 26-35 
Ohki Y, Tabata M, Kuwashima M, Takeuchi H, Nako Y, Morikawa A (2000): Ultrasonographic detection of very thin percutaneous central venous catheter in neonates. Acta Paediatr $\underline{89}, 1381-1384$

Parienti J-J, du Cheyron D, Timsit J-F, Traoré O, Kalfon P, Mimoz O, Mermel LA (2012): Meta-analysis of subclavian insertion and nontunneled central venous catheter-associated infection risk reduction in critically ill adults. Crit Care Med $\underline{40}, 1627-1634$

Parienti J-J, Mongardon N, Mégarbane B, Mira J-P, Kalfon P, Gros A, Marqué S, Thuong M, Pottier V, Ramakers M, et al. (2015): Intravascular Complications of Central Venous Catheterization by Insertion Site. N Engl J Med 373, 12201229

Peters JL, Belsham PA, Garrett CP, Kurzer M (1982): Doppler ultrasound technique for safer percutaneous catheterizatlon of the infraclavicular subclavian vein. Am J Surg 143, 391-393

Pittiruti M, Bertollo D, Briglia E, Buononato M, Capozzoli G, De Simone L, La Greca A, Pelagatti C, Sette P (2012): The intracavitary ECG method for positioning the tip of central venous catheters: results of an Italian multicenter study. J Vasc Access $\underline{13}, 357-365$

Polderman KH, Girbes AR (2002): Central venous catheter use: Part 1: Mechanical complications. Intensive Care Med $\underline{28}, 1-17$

Prekker ME, Chang R, Cole JB, Reardon R (2010): Rapid Confirmation of Central Venous Catheter Placement Using an Ultrasonographic "Bubble Test". Acad Emerg Med 17, e85-e86

Raad I (1998): Intravascular-catheter-related infections. Lancet $\underline{351}, 893-898$

Reynolds N, McCulloch AS, Pennington CR, MacFadyen RJ (2001): Assessment of Distal Tip Position of Long-Term Central Venous Feeding Catheters Using Transesophageal Echocardiology. J Parenter Enter Nutr 25, 39-41

Ruesch S, Walder B, Tramer MRM (2002): Complications of central venous catheters: Internal jugular versus subclavian access-A systematic review. Crit Care Med $\underline{30}, 454-460$

Schummer W, Schummer C, Fritz H (2001): Perforation der V. cava superior bei unerkannter Stenose Fallbericht einer letalen Komplikation einer zentralvenösen Katheterisierung. Anaesthesist $\underline{50}, 772-777$

Schummer W, Schummer C, Schelenz C, Brandes H, Stock U, Müller T, Leder U, Hüttemann E (2004): Central venous catheters-the inability of 'intra-atrial ECG' to prove adequate positioning. Br J Anaesth $\underline{93}, 193-198$

Schummer W, Schummer C, Schelenz C, Schmidt P, Fröber R, Hüttemann E (2005): Optimierte Positionierung zentraler Venenkatheter durch eine modifizierte Anwendung der intravasalen Elektrokardiographie: Validierung mithilfe der transösophagealen Echokardiographie. Anaesthesist 54, 983-990

Schuster M, Nave H, Piepenbrock S, Pabst R, Panning B (2000): The carina as a landmark in central venous catheter placement. Br J Anaesth $\underline{85}, 192-194$ 
Seldinger SI (1953): Catheter Replacement of the Needle in Percutaneous Arteriography: A new technique. Acta Radiol $\underline{39}$, 368-376

Smith RN, Nolan JP (2013): Central venous catheters. BMJ $\underline{347}$, f6570

Strahlenschutzverordnung vom 20. Juli 2001 (BGBI. I S. 1714; 2002 I S. 1459), die zuletzt durch Artikel 8 des Gesetzes vom 26. Juli 2016 (BGBI. I S. 1843) geändert worden ist.

Teichgräber UKM, Gebauer B, Benter T, Wagner J (2004): Langfristige zentralvenöse Zugänge und deren Komplikationsmanagement. Fortschr Röntgenstr $\underline{176}$, 944-952

Vats HS (2012): Complications of Catheters: Tunneled and Nontunneled. Adv Chronic Kidney Dis $\underline{19}$, 188-194

Vezzani A, Brusasco C, Palermo S, Launo C, Mergoni M, Corradi F (2010): Ultrasound localization of central vein catheter and detection of postprocedural pneumothorax: An alternative to chest radiography. Crit Care Med $\underline{38}, 533$ 538

Waldeyer A: Anatomie des Menschen. 17., völlig überarb. Auflage; Walter de Gruyter, Berlin 2003

Walser EM (2012): Venous Access Ports: Indications, Implantation Technique, Follow-Up, and Complications. Cardiovasc Intervent Radiol 35, 751-764

Walshe C, Phelan D, Bourke J, Buggy D (2007): Vascular erosion by central venous catheters used for total parenteral nutrition. Intensive Care Med $\underline{33}, 534-537$

Weekes AJ, Johnson DA, Keller SM, Efune B, Carey C, Rozario NL, James Norton H (2014): Central Vascular Catheter Placement Evaluation Using Saline Flush and Bedside Echocardiography. Acad Emerg Med 21, 65-72

Weekes AJ, Keller SM, Efune B, Ghali S, Runyon M (2015): Prospective comparison of ultrasound and CXR for confirmation of central vascular catheter placement. Emerg Med J 33, 176-180

Weijmer MC, Vervloet MG, Wee PM ter (2004): Compared to tunnelled cuffed haemodialysis catheters, temporary untunnelled catheters are associated with more complications already within 2 weeks of use. Nephrol Dial Transplant $\underline{19}, 670-677$

Wen M, Stock K, Heemann U, Aussieker M, Küchle C (2014): Agitated Saline Bubble-Enhanced Transthoracic Echocardiography: A Novel Method to Visualize the Position of Central Venous Catheter. Crit Care Med 42, e231-e233

Wirsing $M$, Schummer C, Neumann R, Steenbeck J, Schmidt P, Schummer W (2008): Is traditional reading of the bedside chest radiograph appropriate to detect intraatrial central venous catheter position? Chest $\mathrm{J} \underline{134}, 527-533$

Zanobetti M, Coppa A, Bulletti F, Piazza S, Nazerian P, Conti A, Innocenti F, Ponchietti S, Bigiarini S, Guzzo A, et al. (2013): Verification of correct central venous catheter placement in the emergency department: comparison between ultrasonography and chest radiography. Intern Emerg Med $\underline{8}, 173-180$ 
Zeidler K, Arn K, Senn O, Schanz U, Stussi G (2011): Optimal preprocedural platelet transfusion threshold for central venous catheter insertions in patients with thrombocytopenia. Transfusion (Paris) $\underline{51}$, 2269-2276

Zimmermann B (1945): Intravenous Tubing for Parenteral Therapy. Science $\underline{101}$, $567-568$

Internetquellen:

CLABSI Toolkit - Chapter 1 | Joint Commission.

http://www.jointcommission.org/topics/clabsi_toolkit_chapter_1.aspx; Zugriff am 11.12.2016 


\section{Danksagung}

Ein besonderer und herzlicher Dank gilt meinem Betreuer Herrn Dr. med. Peter Korsten für die Konzeption und Vergabe des Promotionsthemas. Mit seiner Hilfsbereitschaft und wissenschaftlicher Kompetenz ermöglichte er die Verwirklichung dieser Dissertation.

Des Weiteren möchte ich mich bei Herrn Prof. Dr. med. Daniel Patschan bedanken für die Durchsicht und Korrektur, der damit maßgeblich zum Gelingen dieser Arbeit beigetragen hat, sowie bei Herrn Prof. Dr. med. Gerhard Anton Müller für die Möglichkeit der Durchführung dieser Dissertation in seiner Klinik.

Ein ganz großes Dankeschön geht an Prof. Dr. med. Volker Ellenrieder, Chefarzt der Klinik für Gastroenterologie und gastointestinale Inkologie, für die stetige Ermutigung und sein Vertrauen in meine Fähigkeiten.

Darüber hinaus möchte ich Herrn David Ellenberger aus dem Institut für Medizinische Statistik der Georg-August-Universität Göttingen für die professionelle Hilfe bei der statistischen Auswertung meinen Dank aussprechen.

Ein ganz großer Dank geht an die ärztlichen Kollegen und das Pflegepersonal der Intermediate Care Station (IMC-Station 1021) sowie der Intensivstation (ICU-Station 1022) der Klinik für Nephrologie und Rheumatologie, die mich liebevoll unterstützt und motiviert haben und ohne deren Mithilfe diese Dissertation nicht realisiert worden wäre. 


\section{Lebenslauf}

Ich, Eirini Mavropoulou, bin am 24.03.1987 als drittes Kind der Eheleute Athanasios Mavropoulos und Angeliki Mavropoulou in Athen (Griechenland) geboren.

Meine Schulzeit begann im September 1992 in Theben. Im Jahre 1998 besuchte ich das Gymnasium mit Ausbildungsrichtung in Mathematik und Naturwissenschaft und legte im Juli 2004 meine Abiturprüfungen mit durchschnittlicher Entlassungsnote ,,ausgezeichnet“ ab.

Im Jahr 2004 habe ich das Studium der Humanmedizin an der AristotelesUniversität in Thessaloniki aufgenommen. Das englische Sprachdiplom Certificate of Proficiency in English, University of Michigan, erwarb ich im Jahr 2007. Das Praktische Jahr habe ich 2009 mit dem ERASMUSAustauschprogramm in der Universitätsmedizin in Göttingen verbracht und die deutsche Kultur kennengelernt. Im Jahr 2010 absolvierte ich das Zertifikat B2 des Goethe-Instituts in Thessaloniki. Nach dem Staatsexamen und dem erfolgreichen Studienabschluss habe ich mein medizinisches Diplom mit der Note „sehr gut" erhalten.

Nach der Einreise nach Deutschland erfolgte am 08.04.2011 die Erteilung meiner Approbation als Ärztin bei der Ärztekammer Niedersachsen. Meine Facharztausbildung für Innere Medizin begann ich am 12.04.2011 in der Abteilung für Gastroenterologie und Endokrinologie im Universitätsklinikum in Göttingen. Seit April bin ich in der Klinik für Gastroenterologie und gastrointestinale Onkologie bei Herrn Prof. Dr. med. Volker Ellenrieder als Assistenzärztin tätig.

Teile dieser Arbeit wurden beim European Respiratory Society International Congress 2015 in Amsterdam vorgestellt und im Supplement des European Respiratory Journal in Abstractform veröffentlicht. 